\title{
A graph for every analysis: Mapping visuals onto common analyses using flexplot
}

\author{
Dustin A. Fife ${ }^{1}$ (D) $\cdot$ Gabrielle Longo $^{2} \cdot$ Michael Correll $^{3} \cdot$ Patrice D. Tremoulet ${ }^{1}$
}

Accepted: 5 December 2020 / Published online: 25 February 2021

(C) The Psychonomic Society, Inc. 2021

\begin{abstract}
For decades, statisticians and methodologists have insisted researchers utilize graphical analysis much more heavily. Despite cogent and passionate recommendations, there has been no graphical revolution. Instead, researchers rely heavily on misleading graphics that violate visual processing heuristics. Perhaps the main reason for the persistence of deceptive graphics is software; most software familiar to psychological researchers suffer from poor defaults and limited capabilities. Also, visualization is ancillary to statistical analysis, providing an incentive to not produce graphics at all. In this paper, we argue that every statistical analysis must have an accompanying graphic, and we introduce the point-and-click software Flexplot, available both in JASP and Jamovi. We then present the theoretical framework that guides Flexplot, as well as show how to perform the most common statistical analyses in psychological literature.
\end{abstract}

Keywords Visualization $\cdot$ Exploratory data analysis $\cdot$ Graphics $\cdot$ Flexplot

"...ifyou assess hypotheses without examining your data, you risk publishing nonsense” (Wilkinson \& Task Force on Statistical Inference, 1999, p. 597)

"A computer should make both calculations and graphs. Both sorts of output should be studied; each will contribute to understanding" (Anscombe, 1973, p. 17)

The current atmosphere in scientific research is one of uncertainty. News of a "replication crisis" is reaching beyond the ears of scientists and into newspapers and public discourse. Additionally, the frequency of article retractions is growing (see www.replicationwatch.com), and with it, greater awareness of scientific mistakes and misconduct. While these conditions invite a growing unease among scientists, it is also a time of great opportunity, where change comes easier as researchers recognize the need for an evolution in the methodological status quo.

This is not the first time psychology has undergone a methodological upheaval. In the 1990s, many researchers were up in arms, calling for journals to ban the use of $p$ values (e.g.,

Dustin A. Fife

fife.dustin@gmail.com

Rowan University, Psychology, Glassboro, NJ, USA

University of Houston, Houston, TX, USA

Tableau Research, St. Seattle, WA, USA
Schmidt, 1996). Though governing bodies assembled task forces, and made recommendations (e.g., Appelbaum \& Cramer, 1974; Wilkinson \& Task Force on Statistical Inference, 1999), little changed after this upheaval (Counsell \& Harlow, 2017; Cumming et al., 2007). Among these recommended changes was a call for the greater use of data visualization, which has, sadly, been unheeded in psychology (Kyonka, Mitchell, \& Bizo, 2019).

This was not the first revolution where the call for greater visuals was made and ignored. Only a few decades ago, many scientists were analyzing data by hand. Since the advent of computers, crunching numbers went from a laborious process to one that requires little thought. Using only a few clicks, one can have pages upon pages of tables, statistics, $p$ values, effect sizes, etc. With increased computational power, analyses and the very questions scientists can ask also have become more sophisticated.

Yet some have expressed disappointment in how this computer-inspired revolution has evolved, particularly with graphical data analysis. Wainer (2010) noted,

“...thirty years ago I was enthusiastic and optimistic about the future of graphical use. I thought software would be built with sensible default options, so that when the software was set on maximal stupidity (ours not its), a reasonable graph would result...Alas, I couldn't have been more wrong. Instead of making wise, evidence-based choices, default options seem to 
have been selected by the same folks who deny landing on the moon, global warming, and evolution..." (Wainer, 2010, p. xi, in Bertin, 2010).

We agree, at least with the most popular statistical software platforms used in psychology. ${ }^{1}$ While there are some programs with light coding requirements and sensible defaults (D3, VegaLite, Spotfire, Tableau, Power BI, etc.), these programs and the visualization principles they follow are not well adopted in psychological (or biomedical) research. For example, past surveys of psychological research show that both textbooks (Butler, 1993; Peden \& Hausmann, 2000) and journal articles (Kyonka et al., 2019; Schild \& Voracek, 2013; Weissgerber, Milic, Winham, \& Garovic, 2015) rely heavily on graphics that bias human perception, e.g., bar graphs and line graphs. Representing means using bar graphs leads people to believe values within the bar are more likely than values above the bar, even for symmetric distributions (Zacks \& Tversky, 1999). Also, because the bottoms of bar graphs fall at $y=0$, they give the false impression negative values are impossible, which may lead some to underestimate the variability in data. Furthermore, some statistical programs (e.g., Excel), make it extremely difficult or even impossible to produce standard graphics (e.g., violin plots, boxplots, or even histograms). Other programs (e.g., SPSS) make it difficult or impossible to produce graphs that follow the practices we outline for data visualization, such as plots with raw data when group means are plotted. Still others (e.g., R) have such a steep learning curve that only the most savvy of researchers even attempt to pilot its code. In nearly all cases, creating visuals is ancillary to the analysis; individuals must perform additional steps, often clicking through deeply nested menus in order to produce a graphic (Hu, Orghian, \& Hidalgo, 2018). All of these reasons lead to massive incentives to utilize misleading, suboptimal visualizations or produce no graphics at all.

While the computational revolution leapt ahead of any sort of graphical revolution in psychology, we hope visualization is not forgotten in the post-replication crisis transition. Indeed, visuals offer many advantages over numerical summaries. First and foremost, visual information can be understood in fractions of a second, while the same information presented as a table would take much longer to interpret (Hansen, Chen, Johnson, Kaufman, \& Hagen, 2014). Consider the table/ image shown in Fig. 1. The table contains the same information as the image, but the image conveys that information visually. Which is easier to understand?

In addition to enabling the rapid understanding of information, visuals also provide a critical quality-control check. Suppose, for example, we were interested in how PTSD

\footnotetext{
${ }^{1}$ One reviewer suggested that it's not software defaults that are the problem. Rather, it is our discipline's focus on means, mean difference, ANOVAs, etc., that is largely to blame; these procedures quite strongly emphasize means, and so the visuals reflect that emphasis.
}

$\begin{array}{cc}\text { Hours Studied } & \text { Exam Score } \\ 0.1 & 85 \\ 0.2 & 86 \\ 0 & 45 \\ 0 & 62 \\ 0 & 66 \\ 0.1 & 85 \\ 0 & 75 \\ 0.3 & 86 \\ 0.2 & 84 \\ 0.3 & 87\end{array}$

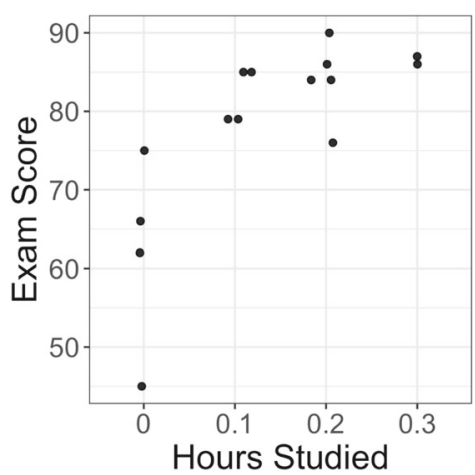

Fig. 1 The table and the image both contain the same information, but the image conveys that information graphically, which improves encoding

symptoms differed between male and female combat veterans. Further suppose that gender was recorded as a number in the survey (e.g., $1=$ Female, 2 = Male), yet we mixed up the labels when performing data analysis. If we computed a $t$ test, we might conclude females suffer more severe PTSD than males $(t[1163]$ $=6.86, p<0.001, d=1.21)$. This would be a false conclusion we might easily make. Yet a graphic (Fig. 2), which displays raw data with 25 th $/ 50$ th $/ 75$ th percentiles, readily shows there are many more females than males who served in combat. This graphic, one would hope, should invite much-needed suspicion and reveal the error. The ability of visualizations to act as "sanity checks" for data (Correll, Li, Kindlmann, \& Scheidegger, 2019) has made them a crucial part of both academic and commercial data cleaning tools, such as Profiler (Kandel, Parikh, Paepcke, Hellerstein, \& Heer, 2012).

Another advantage of graphics is that they provide an easy means to determine whether the statistical model actually fits the data. Or, if we were to take this one step further, visuals are simply another representation of a statistical model, beyond, for example, a regression equation. Consider the image shown in Fig. 3, which shows the relationship between the number of possessions worth more than $\$ 1,000$ and stress. A standard regression model (shown in red) assumes a linear relationship. However, the actual relationship is better described by a quadratic function (blue line). The two models offer very different theoretical interpretations of the relationship between possessions and stress. The red model suggests increasing possessions always reduces stress, while the blue (more appropriate) model shows that, at some point, increased possessions are associated with more stress. Once again, there would be no way to determine this from summaries of data. With a graphic, on the other hand, that information is conveyed easily and strikingly. Visuals are the best (and sometimes only way) to verify our statistical models actually make sense.

\section{The importance of visualization accessibility}

In an ideal world, all of the aforementioned problems would be solved by researchers adopting $\mathrm{R}$ and ggplot2 (Wickham, 


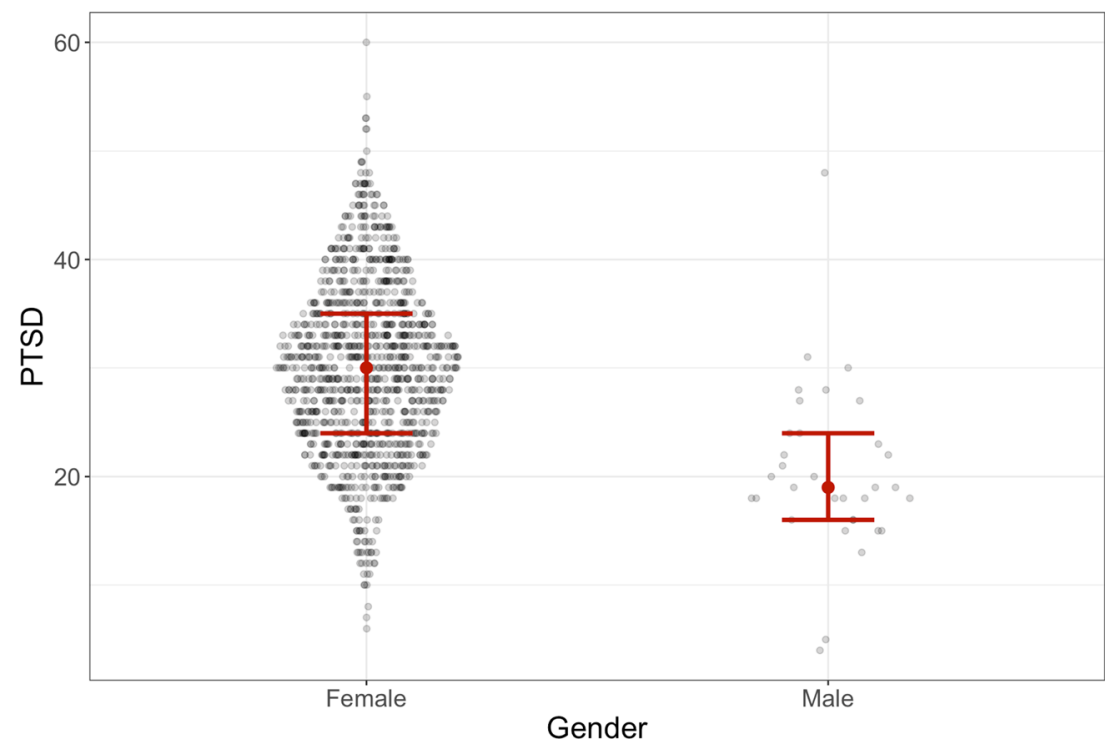

Fig. 2 This graphic allows for a quick quality control check. Each dot represents an individual score. The center dot shows the median, and the upper and lower limits of the bars are the 75th and 25th percentiles, respectively. Notice that the graph indicates there are more female

2016), an R package devoted to visualizations. Unfortunately, market share for SPSS is at least double that of R, according to Google Scholar citations (Muenchen, 2019), though that number is declining. The problem with $\mathrm{R}$ is the learning curve is quite steep. For scholars already struggling to balance demands of teaching, research, grant writing, service, etc., having to learn a programming language and change the way in which they engage in research is a monumental task.

Even if researchers were to adopt $\mathrm{R}$ en masse, there is yet another learning curve they may need to overcome for sound visualizations: ggplot2. ggplot2 is a powerful tool that offers infinite flexibility in designing visualizations. Also, there is a large community of ggplot2 users willing and able to assist new users in the nuances of graphics. However, though ggplot2 is technically an R package, the "grammar of graphics" combat veterans than male veterans. This contradicts intuition and would make it less likely someone would make a data analysis error. A visualization of just the mean and error would not make this miscategorization visible

(Wickham, 2010) for ggplot 2 is quite different from the grammar of R. For this reason, even sophisticated R users may be reluctant to adopt ggplot2. Furthermore, while ggplot2 is extremely flexible, it can often require multiple lines of code to generate simple graphics (Fife, 2020a). Finally, producing graphics with ggplot2 is ancillary to data analysis; it's an additional step researchers may be too exhausted to take, and there is no guarantee these graphics will accurately represent the statistical models.

Flexplot resolves all these difficulties. For experienced $\mathrm{R}$ users, Flexplot offers a friendly interface for interacting with ggplot2, reducing the lines of code required to produce sound visualizations. Furthermore, Flexplot makes it easy to visualize statistical models; users only need to wrap statistical models (e.g., $1 \mathrm{~m}$ models in R) in the visualize function to automate visuals of statistical models.

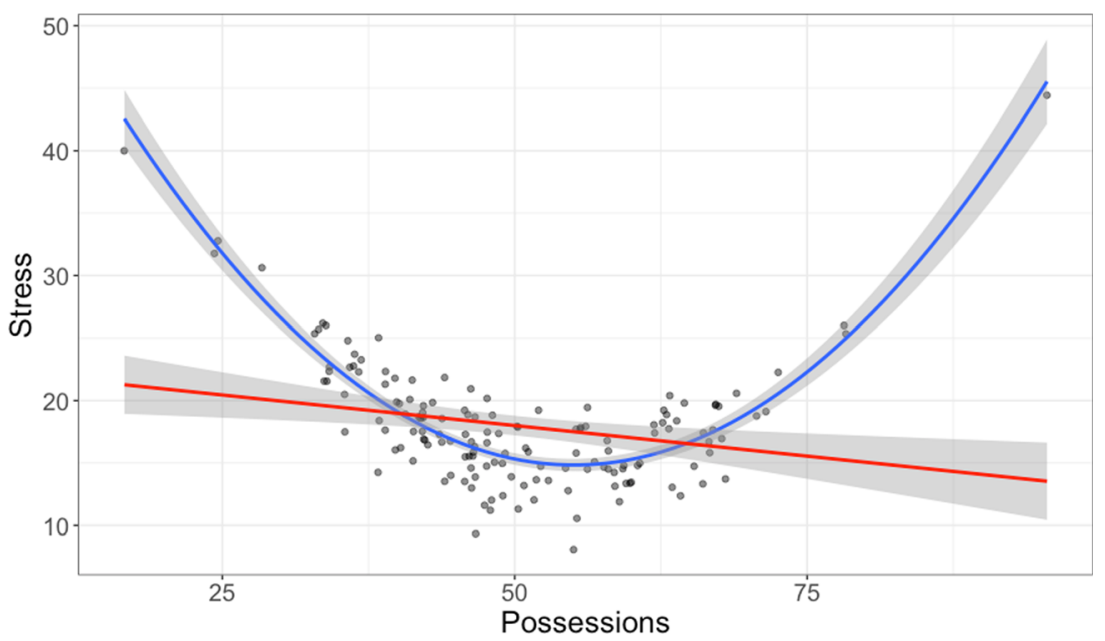

Fig. 3 Standard regression line (in red) and a quadratic line (blue). Graphics reveal that the original regression line poorly models the data 
For those uncomfortable with the SPSS-to-R leap, nearly all of Flexplot's functionality can be utilized in the point-andclick software of JASP and Jamovi. In addition to providing sound visualizations, the JASP/Jamovi versions of Flexplot further integrate modeling with visualizations, which we will illustrate throughout this paper.

In summary, sound visualization is part and parcel of sound statistical modeling, yet the existing tools either cannot do sound visualizations, they require a great deal of effort to utilize these visualizations, or visualization is ancillary to data analysis. In this paper, we introduce new visualization software called Flexplot, which was designed for psychologists. Its purpose is to make statistical graphics intuitive to produce and intuitive to interpret.

We begin by explaining how Flexplot was designed to pair statistical modeling with visualization. We then explain the basic philosophy behind Flexplot, beginning by conceptualizing data visualization from a human factors perspective. We then demonstrate how to create graphics for common statistical models in an easy-to-use graphical interface. Throughout, we demonstrate how to pair visualization and modeling to maximize the quality of interpretation. We then conclude with recommendations and resources available to researchers to allow them to utilize visuals in the post-replication crisis revolution.

\section{Flexplot's philosophy: A human factors approach}

Software has become the tool of choice for summarizing data and, as such, plays a critical role in the scientific process. Statistical software guides our interpretation. Unfortunately, statistical software can bias interpretation in unintentional ways (Pandey, Rall, Satterthwaite, Nov, \& Bertini, 2015; Zacks \& Tversky, 1999). Likewise, statistical software can also stand in the way of meaningful interpretation by creating unnecessary obstacles.

Human factors (HF) examines the relationship between human beings and the systems with which they interact (Kohn, Corrigan, \& Donaldson, 2000). One of the goals of $\mathrm{HF}$ is to design work tools, such as software and other technologies, to maximize productivity, effectiveness and worker health and safety (Adams, 1989). According to these principles, technology should facilitate the pursuit of the goals users intend to accomplish (Vicente, 2010). For statistical software, that means the software should make it easy to make sense of data. Likewise, technology needs to minimize misinterpretation (Lee, Blanco, $\&$ Bo, 2017). If software is poorly constructed, it will impede accurate interpretation of data and possibly even invite confusion or misconceptions.
The design of Flexplot was guided with human factors principles in mind. More specifically, Flexplot abides by three guiding principles:

1. Design graphics that leverage human strengths and minimize human biases.

2. An appropriate graphical representation should accompany every statistical analysis.

3. Minimize obstacles to producing graphics.

We will discuss each of these in turn.

\section{\#1: Design graphics that leverage human strengths and minimize human biases}

Various guidelines exist for designing visualizations. These are often built on existing work on graphical perception and vision science (Munzner, 2014; Ware, 2019). Central to the design of visualizations is the recognition that not all visual encodings of data are equally useful or accurate for analytical tasks (Cleveland \& McGill, 1984). For example, certain visual channels such as position on a common axis afford greater precision in decoding values than channels such as color hue or value.

For the purpose of visualizing statistical models, plots are often concerned with syncretic/aggregate tasks, like assessing central tendency or spread (Healey, Booth, \& Enns, 1996; Szafir, Haroz, Gleicher, \& Franconeri, 2016). Because many statistical models rely on inferring general principles from aggregate information, visuals that encode such aggregate information should be designed in such a way that they are easily encoded. Designing visuals for ease in performing these sorts of "visual statistics" (Correll, 2015) is qualitatively different than designing for the decoding of individual values.

The principles of "expressiveness" and "effectiveness" of visualizations (Mackinlay, 1986) are particularly important for aggregate encoding. These principles, instrumental to other automated charting regimes such as APT (Mackinlay, 1986) and Tableau's ShowMe (Mackinlay, Hanrahan, \& Stolte, 2007), similarly guide Flexplot. Expressiveness holds that a visualization should only "encode the data relations intended and no other data relations" (Card, 2009, p. 195). In other words, the visualization should contain what is appropriate for the human task and no more (Norman, 2014). The expressiveness principle is similar to the position advocated by Tufte (Tufte \& Graves-Morris, 1983), that one should eliminate "chart junk" and aim for maximizing the data to ink ratio (see also Inbar, Tractinsky, \& Meyer, 2007).

In addition to appropriate minimalism, effectiveness also implies a visualization should be designed to support the capacities of the human visual system: we should refrain from using graphics where we expect the user to be biased or misled (Dimara, Franconeri, Plaisant, Bezerianos, \& Dragicevic, 
2018). For example, bar plots of means suffer from a "within the bar bias," which means people tend to perceive values below the top of the bar as more likely than values above the bar, even when the underlying distributions are symmetrical (Correll, 2015; Correll \& Gleicher, 2014; Newman \& Scholl, 2012). Likewise, we should avoid graphs which utilize slower or less accurate perceptual systems (Cleveland \& McGill, 1984). For example, Chernoff faces (Chernoff, 1973) are both slower and less accurate than traditional spatial representations of data (e.g., Lee, Reilly, \& Butavicius, 2003).

For these reasons, Flexplot defaults to utilizing simple scatterplots for most visuals, including visuals which display group scores on a numeric outcome (e.g., in a graphic used to represent results from a $t$ test). These visuals rely heavily on positional perceptual channels, which are known to be highly sensitive to detecting differences (Cleveland \& McGill, 1984). Flexplot also attempts to embed as much information as is necessary to understand the data, and no more. A typical graphic from Flexplot will display central tendency, spread, range, sample size, and distribution density, all in the same graphic.

While there are many heuristics and/or principles of visualization (e.g., Kosslyn, 2006; Moritz et al., 2018; Tufte \& Graves-Morris, 1983) and display design (Lee et al., 2017), there are few hard and fast rules. Almost all "rules" have exceptions (e.g., never use pie charts, Tufte \& Graves-Morris, 1983, except when one's task is to judge proportions, Hollands \& Spence, 1998; see Kosslyn, 2006 for a review). Also empirical evidence for one visual versus another are often sparse or contradictory (e.g., the principles of "redundancy" versus "clutter," Lee et al., 2017). It seems that there are a wide range of visualization strategies that may be effective at conveying statistical information, and one's choice of visualization strategy may be guided by both taste and general visualization principles. It is no different for Flexplot; the overall design is guided by the principles of expressiveness and effectiveness, but in situations where the literature is ambiguous or unknown, Flexplot's defaults are guided by both aesthetics and principles of statistics. Throughout this paper, we will highlight some of those decisions and the rationale behind them.

\section{\#2: An appropriate graphical representation should accompany every statistical analysis}

As mentioned previously, visuals provide an easy method of diagnosing problematic statistical models and rapidly interpreting statistical information that can be missing from reports of the analyses or summary statistics per se (Anscombe, 1973; Matejka \& Fitzmaurice, 2017). They also can natively convey information about variability. Also, visuals allow audiences to make informed decisions about the results of statistical analyses themselves, rather than relying on normed (and arbitrary) benchmarks like those for statistical significance.
For these reasons, many scholars have advocated visuals should be heavily used in quantitative research (Healy \& Moody, 2014; Levine, 2018; Tay, Parrigon, Huang, \& LeBreton, 2016; Wilkinson \& Task Force on Statistical Inference, 1999). Some (e.g., Fife, 2020b) go so far as to say every analysis should have a graphic, a sentiment which we echo and extend. Doing so not only protects analysts from publishing nonsense (Wilkinson \& Task Force on Statistical Inference, 1999), but also allows audiences to make informed judgments about the results of a statistical analysis (Fife, 2020b; Healy \& Moody, 2014; Tay et al., 2016).

When utilizing visualizations for statistical modeling, it is also critical to show raw data, rather than simply visualizing summaries of raw data (e.g., a regression line or standard error bars; Fife, 2020b). The rationale behind this is twofold. First, when users are able to plot raw data, they can better evaluate the degree to which the model fits (Levine, 2018). For example, one can determine, at a glance, whether outliers are driving the value of the mean, whether a curvilinear relationship is better suited than a linear one, and/or whether critical assumptions are violated. Second, raw data better convey uncertainty than summaries of statistics. ${ }^{2}$ One way in which they do so is by preserving the scale of the axes, which has been shown to better communicate uncertainty about the size of treatment effects (Hofman, Goldstein, \& Hullman, 2020). Also, raw data better communicate uncertainty by highlighting deviations from a model's fitted values (e.g., a regression line or group mean).

\section{\#3. Minimize obstacles to producing graphics}

As we mentioned previously, visualizations are too often ancillary to data analysis; tables and test statistics are prominently displayed, while visuals, if they are produced at all, are rarely published (Kyonka et al., 2019). Flexplot automates model visualization and places visuals prominently, making them the first thing analysts see when fitting statistical models. ${ }^{3}$ Doing so minimizes obstacles to graphical data

\footnotetext{
${ }^{2}$ Another alternative to raw data is to display hypothetical outcome plots (HOPs). These animated plots simulate draws from distributions for, e.g., treatment and control groups. These better communicate uncertainty than simple means \pm standard errors (Hofman et al., 2020), though there's some evidence these plots don't have an advantage over static displays of uncertainty (Kale, Kay, \& Hullman, 2020).

${ }^{3}$ Clearly, one of the important tasks relegated to Flexplot is to determine the variable type, as variable type will dictate the sort of graphic chosen. When using Flexplot in R, it will make this determination by identifying whether the variable is a factor/character, ordinal factor, or a number. Occasionally, Flexplot will make an error (e.g., when a number represents an ordinal or nominal variable). Within $\mathrm{R}$, the user simply needs to convert the variable to the appropriate type (e.g., character, ordered factor, factor). Likewise, in Jamovi/JASP, the user can change the data type to nominal or ordinal. Unfortunately, both JASP and Jamovi default to treating integers with less than 20 unique values as ordinal variables, which can cause some problems for visualizations. In this case, the user simply needs to convert the variable type through the data interface.
} 
analysis. For this automation to happen, it is important to conceptualize standard statistical models from the linear model perspective.

\section{The linear model approach}

Most statistical methods taught in introductory textbooks are subsumed by the general linear model (GLM), which is essentially regression (Cohen, 1968). The majority of statistical procedures can be re-expressed as GLMs. For example, to perform a one-sample $t$ test, one can fit an intercept only model, then test whether that intercept is different from the value of interest. Likewise, an independent groups $t$ test can also be re-expressed as regression. To do so, we could label one group (e.g., the control group) as a zero, then label the treatment group as a one. If we then fit a regression model (i.e., GLM), the intercept is the mean of the control group and the slope is the difference between the treatment and control group.

The advantage of conceptualizing statistical models as a GLM is practical: rather than having to memorize complex decision trees to arrive at the appropriate analysis, one simply has to specify the predictor variable(s) and the outcome variable and the computer handles the $t$ test (or ANOVA or factorial ANOVA, etc.) to regression conversion in the background. Cognitive scientists have long known there are limits on the amount of information we can process, and we can often "hack" our capacity by "chunking" information, which amounts to lumping distinct elements into groups (Baddeley, 1994; Miller, 1956). In a sense, conceptualizing statistical models as linear models is a means of "chunking" distinct elements (e.g., ANOVA, factorial ANOVA, onesample $t$ test, independent $t$, regression, etc.) into one, which assists in transferring information from short-term into long-term memory.

Typically, we tend to talk about categorical independent variables having "mean differences" on the dependent variable, while we say numeric predictor variables "predict" or "explain" the outcome variable. We could just as easily state that a categorical variable (e.g., group assignment) "predicts" or "explains" scores on the outcome variable. The difference in semantics is psychological, not mathematical. Likewise, conceptualizing grouping variables (e.g., treatment versus control) as "predictor" variables makes no difference for the mathematics; math doesn't care whether we say a variable predicts an outcome or that there are mean differences on the outcome for the grouping variables. However, conceptualizing them as predictor variables makes the categorical versus numeric distinction less important (as it should be) and helps the analyst focus instead on interpreting data, rather than choosing the "right" analysis. In other words, the GLM approach saves mental resources, leaving more room to invest those resources in interpreting the analysis. ${ }^{4}$

Flexplot takes a similar approach. The user need not choose between a boxplot versus scatterplot versus histogram versus bar chart. Rather, the user simply specifies the variable(s) they wish to visualize and the computer handles the decision-making in the background. (Similar efforts to automate visualization have been attempted. See Jun et al., 2019). ${ }^{5}$

\section{Accessing Flexplot}

The first author developed Flexplot as an $\mathrm{R}$ package (Fife, 2020a) beginning in 2018. In August 2019, he developed a module for Jamovi (The Jamovi Project, 2019), then in February 2020, he developed a similar module in JASP (JASP Team, 2019) called "Visual Modeling." In this paper we will illustrate the software in the JASP module, though the Jamovi module behaves similarly.

Within the Visual Modeling module in JASP, there are four submodules (see Fig. 4). The first (called Flexplot) is dedicated exclusively to plotting. As such, it is very flexible in producing graphics, but does not produce statistical estimates. The other three submodules (Linear Modeling, Mixed Modeling, and Generalized Linear Modeling) pair statistical modeling with visualization. As such, they do produce statistical estimates, but are less flexible than the Flexplot module in controlling how variables are displayed.

We begin by introducing the Flexplot submodule. We then spend the rest of the paper demonstrating the Linear Modeling submodule. We do not go into detail with the other two submodules (Mixed Modeling and Generalized Linear Modeling), mostly because these sorts of analyses are less common. Also, our introduction to the Linear Modeling submodule will likely provide enough of a foundation that users could easily utilize the other two modules with little to no guidance. For those wishing more direction, see the following tutorial on JASP's website: https://jasp-stats.org/2020/ 04/21/the-visual-modeling-module/.

\footnotetext{
${ }^{4}$ Multiple resource theory (Wickens, 2008) states that dividing information across resources (visual vs auditory vs tactile) can enable more information to be understood. A corollary of this theory is that too much use of a single type of resource will result in a bottleneck or error. Presumably, choosing the "right" analysis and interpreting the data both tap into the same type of cognitive processes, so reducing the need for the first leaves more capacity for the latter. See also Kantowitz (1987).

${ }^{5}$ There is a natural tension between flexibility/user control and ease of use/ automation. Flexplot attempts to balance this tension by utilizing visualization principles to produce default graphics, while allowing users to easily modify the defaults.
} 


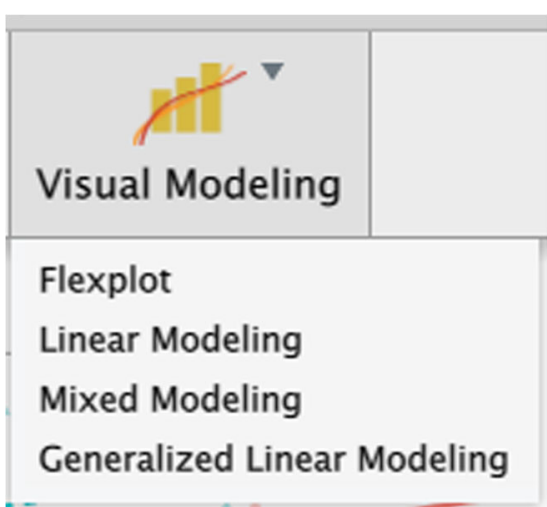

Fig. 4 The Visual Modeling module in JASP. This module has four "submodules" designed to assist with visualizing statistical models. The first (Flexplot) is dedicated to graphing. Linear modeling is for fitting linear models (e.g., regressions, $t$ test, ANOVAs). The second, Mixed Modeling, is for fitting mixed models. The last, Generalized Linear Modeling, is for fitting generalized linear models (e.g., logistic regression, Poisson regression)

\section{Data visualization: the Flexplot submodule}

Because the Flexplot submodule is dedicated exclusively to plotting, it offers more control over how variables are displayed. In both Jamovi and JASP, Flexplot has a box on the left that identifies all the variables in the dataset (see Fig. 5). It also has three boxes on the right: Dependent Variables, Independent Variable(s), and Paneled Variable(s). Flexplot will display the variable placed in the "Dependent Variable" box on the $Y$-axis (except when no independent variables are specified, in which case, Flexplot will display a histogram for numeric variables and a bar chart for categorical variables). Flexplot will display the first variable specified on "Independent Variable(s)" on the $X$-axis. If the user specifies a second variable, Flexplot will display that variable as different colors/symbols/lines. The software only allows a maximum of two variables in the "Independent Variable(s)" box; if users wish to visualize additional variables, they must use the "Paneled Variable(s)" box as well.

The "Paneled Variable(s)" box controls, not surprisingly, paneling. When a variable is included in this box, the software will generate different scatterplots for different levels of the variables specified. For example, if the user places "gender" in the Paneled Variable(s) box, Flexplot will generate a scatterplot for males, a scatterplot for females, etc. The first variable entered will have different plots listed in different columns. The second variable entered will have different plots listed in different rows.

Figure 6 shows four plots generated by Flexplot. The top left shows what happens when only one variable (a numeric variable) is placed in the "Independent Variable(s)" box. The second shows what happens when two are placed there. The third and fourth show what happens when paneled variables are specified. Note: the top-right image has a great deal of overlap between datapoints, which makes it difficult to interpret the results. While Flexplot attempts to minimize confusion by providing the additional cues (Lee et al., 2017) of shape and line type, it can still be difficult to distinguish between the two groups. For this reason, we recommend paneling before overlaying colors/symbols/lines on the same graph. However, this image is merely to show how the second variable in the "Independent variable(s)" box is displayed.

In the dataset used to generate these images, both IQ and agility are numeric variables, yet they are displayed as if they have three distinct groups. In the background, if the user specifies numeric variables in the panels (or even as a second independent variable), Flexplot will "bin" the variable into distinct levels using quantiles (e.g., 0-33\%, 33-66\%, 66-100\%). It uses quantiles (rather than, e.g., separating using standard deviations) because quantiles ensure equal sample sizes across bins. If one were to use standard deviations and had skewed data, it is quite possible to have bins with very different sample sizes. Furthermore, while some visualization techniques (e.g., linear regression) are insensitive to how many datapoints are contained within a bin (because the regression model is fit to the entire dataset, rather than just the data within a bin), some visualization techniques (e.g., loess lines) are explicitly fit for just the data within a bin. If one bin had a small sample size, the loess line would be quite unstable. Utilizing quantiles to bin thus ensures maximal precision within each bin.

Not only can the user control how variables are represented in a graphic, but they can also control other features of the graphic, including the transparency of the datapoints, the type of intervals displayed (e.g., quartiles, the default, versus standard errors versus standard deviations), and whether confidence bands are displayed.

One option that deserves special mention is the use of ghost lines, which make it much easier to interpret paneled graphics. While panels allow users to visualize complex multivariate relationships, they also make it harder to make comparisons across panels. For example, which slope is steeper in the left image of Fig. 7, the placebo slope or the treatment B slope? To determine that, the eye must travel back and forth quite a distance to make a judgment. When doing so, there is a cost (in time and effort) associated with shifting attention across spaces (Wickens, 2014). If, however, we were to overlay the line from the placebo group to Treatment B group as in the right image, it makes it very easy to see that Treatment B group has a steeper slope, and is consistent with the human factors display design principle of "minimizing information access cost" (Lee et al., 2017). We call the red line a "ghost line. ${ }^{\prime 6}$ In JASP, the ghost line will repeat the pattern from the

\footnotetext{
${ }^{6}$ The first author of this paper created the name "ghost line" after the concept of racing "ghosts" in racing video games. These racing ghosts are transparent recordings of a player's previous performance that are displayed during a race. They do not interact with the players of the race, they merely provide a reference of the previous player's performance. Apparently, a similar concept seems to have been invented by Lee et al., 2017, though they called these lines "visual landmarks."
} 


\section{Flexplot (Graphics)}

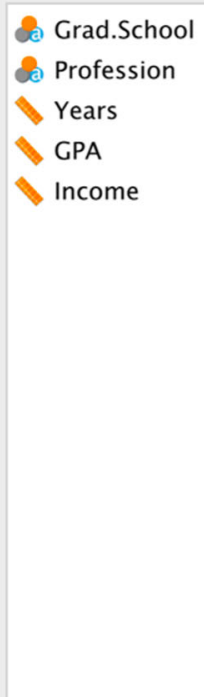

$+\mathrm{i} \times$

Dependent Variable

Independent Variable(s)

Panelled Variable(s)

Fig. 5 The user interface for the Flexplot submodule in the Visual Modeling module in JASP

middle-most panel. Future releases of JASP will allow the user to control from where the ghost line originates.

In summary, the variable specified in the Dependent Variable box will be on the $Y$-axis, and the variables specified in the Independent Variable(s) box will be displayed first on the $X$-axis, then as different colors/symbols/lines. The variables specified in the Paneled Variable(s) box will create panels in the rows (for the first variable entered) and in the
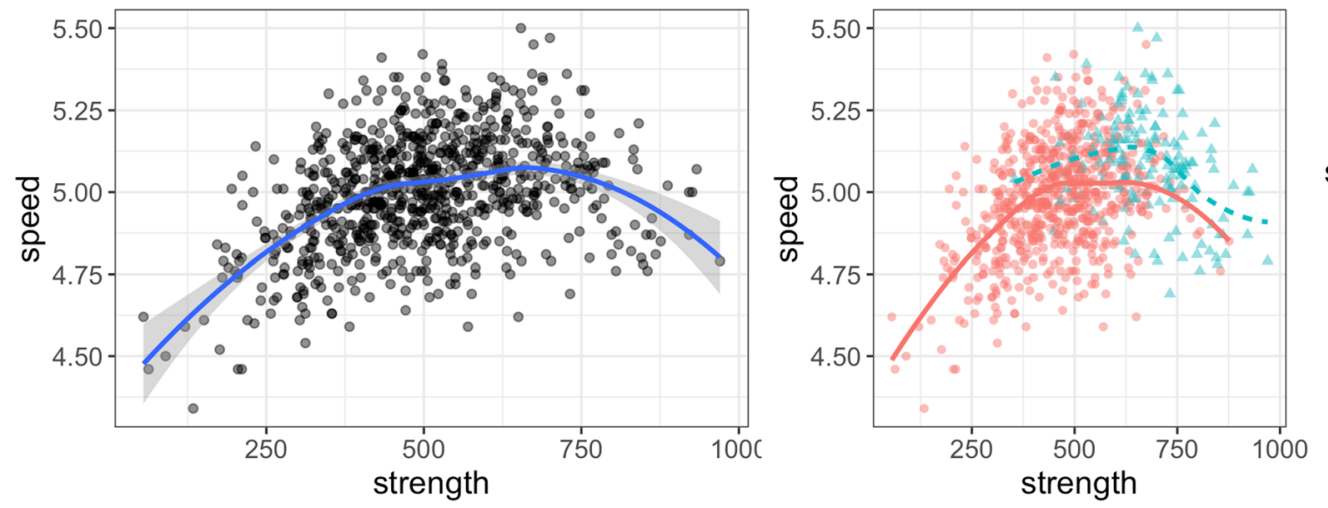

superpower

- no

$=-$ yes
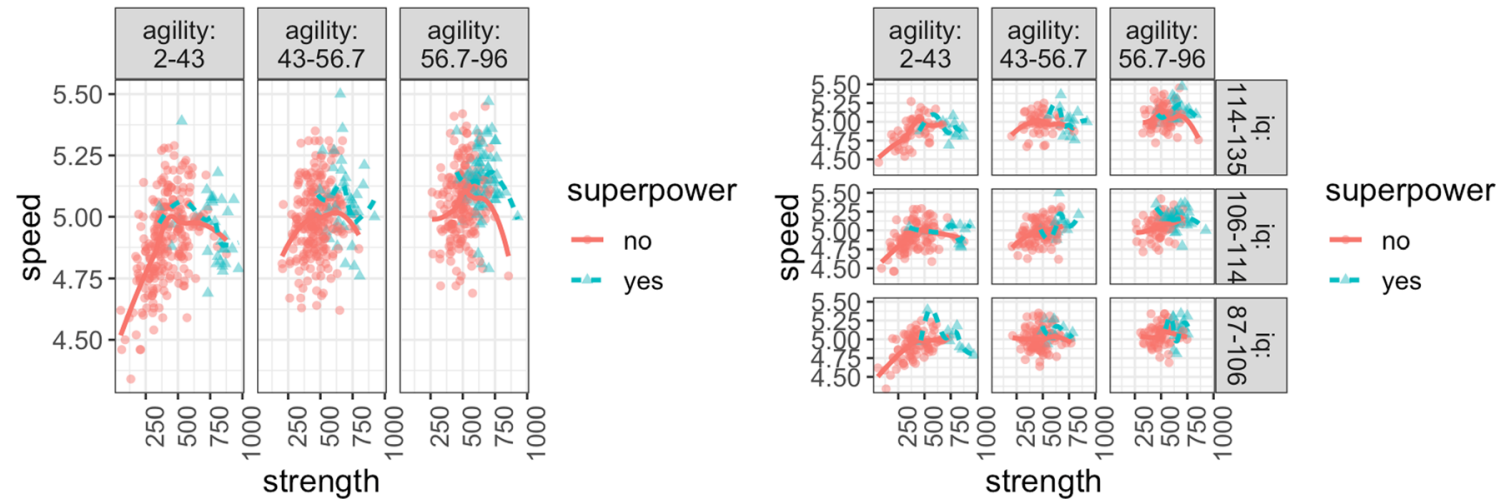

Fig. 6 Four different graphics produced by Flexplot. The first has only one independent variable specified, while the second (top right) has two specified. The third/fourth (bottom rows) show what happens when the user specifies paneled variables 

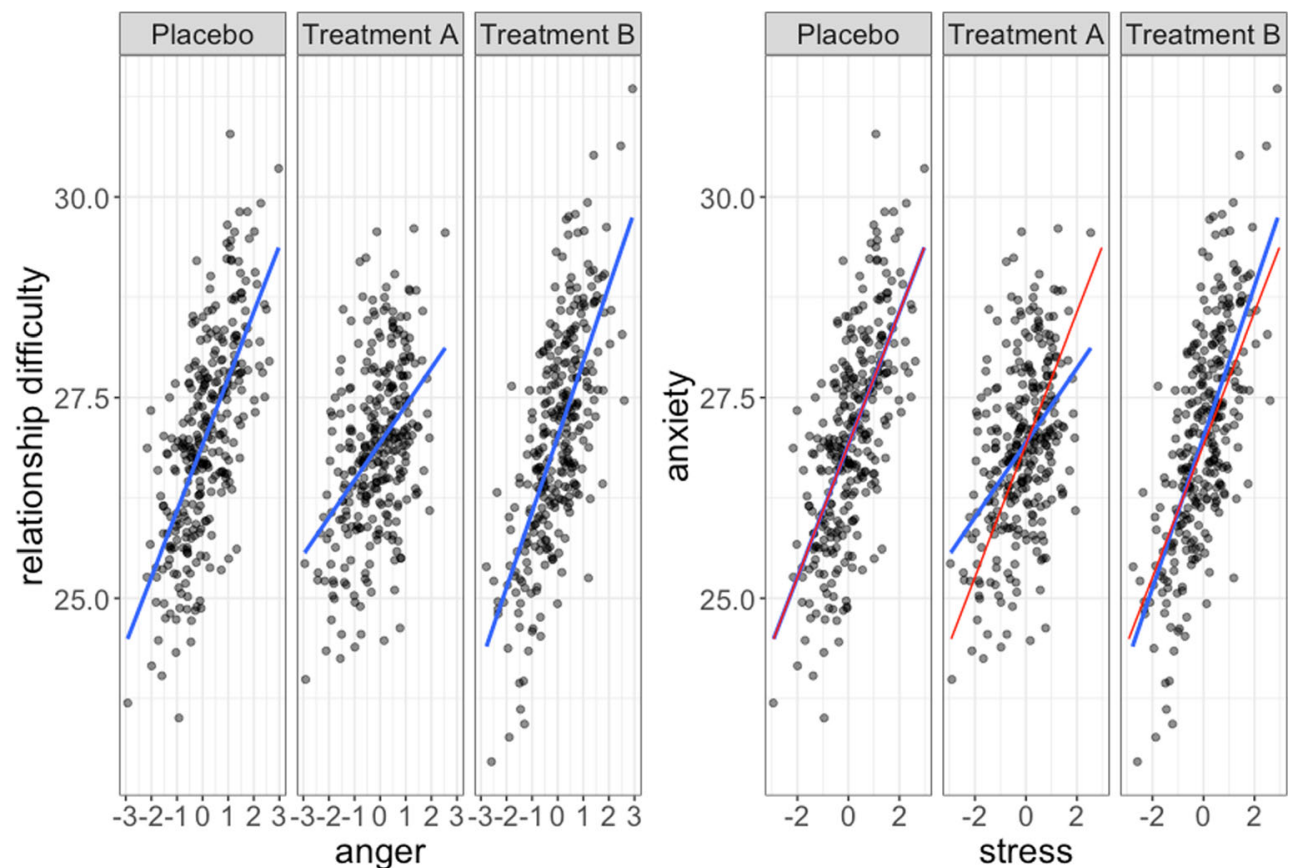

Fig. 7 Two figures of the same data. The right image displays red "ghost lines," which repeat the pattern from the placebo condition to the other conditions. This makes it easier to detect differences in slopes between conditions

columns (for the second variable entered). When paneling, the user can ask the computer to overlay ghost lines to make it easier to compare across panels.

\section{Pairing statistical modeling with visualization: the linear modeling submodule}

As we mentioned previously, one of the guiding principles of Flexplot is to minimize obstacles to producing graphics. The three modeling modules in JASP (Linear Modeling, Mixed Modeling, and Generalized Linear Modeling) automate visualization whenever the user fits a statistical model. Using the Linear Modeling submodule, the analyst simply specifies an outcome variable and one or more predictor variables. (Mixed models and generalized linear models may require additional specification). In the background, Flexplot automates the visuals. Some of the rules for how Flexplot decides on visuals will be explained throughout this paper (e.g., whether to produce a scatterplot versus another type). For multivariate data, the automation is more complex. In short, with multivariate data, it tends to plot numeric variables on the $X$-axis and tries to avoid plotting any variable as a separate line/color/symbol, simply because that increases overlap between datapoints and makes it more difficult to interpret graphics. If it can't be avoided, Flexplot represents the categorical variable with the least number of levels as separate lines/colors/symbols (again, to minimize overlap). If the analyst is not pleased with how the visual is represented, they can always use the Flexplot submodule for more control.

In the following sections, we will explain both how to visualize common statistical models, as well as explain the rationale behind these graphics. While Flexplot is based on a general linear model approach, we will still illustrate common statistical models using traditional nomenclature (e.g., $t$ test, ANOVA, regression).

\section{Simple regression}

A regression model is utilized when one has a numeric predictor and a numeric outcome. The standard approach to visualizing numeric predictor/outcome data is a scatterplot. Flexplot follows the same convention, since scatterplots are intuitive, easy to interpret, and commonly used.

To perform a simple regression in the Linear Modeling submodule, the user simply specifies the outcome of choice and a numeric predictor variable. Subsequently, the computer will display a scatterplot with the raw data and the regression line will be shown in red. Also, by default the software will report an intercept, slope(s), standardized slope(s), and 95\% confidence intervals for all estimates. It defaults to not report $p$ values. In light of the evidence $p$ values bias human perception in unintentional ways (Gigerenzer, 2004; Oakes, 1986; Tyron, 1998), the first author elected to make them far less visible in the Visual Modeling module. (The user can click on "Show $p$ values" under the "Results Display" menu to report $p$ values). 
The user can also ask for univariate distributions, which, for simple regression models, will display two histograms (one for the predictor, one for the outcome). The user can also request diagnostic plots, which evaluate model assumptions. Checking this button will display a histogram of the residuals (to assess the normality assumption), a scale-location (SL) plot (to assess the homoscedasticity assumption), and a residual dependence plot (to assess the linearity assumption). For a tutorial on utilizing visuals for diagnostics, see http:// quantpsych.net/stats modeling/diagnostics.html, as well as a demonstration by Fife (2020b).

Other options include the ability to omit the reporting of confidence intervals and slopes/intercepts. The "Model comparisons" option allows users to perform model comparisons on nested models (Rodgers, 2010). For regression, the "full" model is the model of interest, and the "reduced" model is a mean model. Two other options ("Report means" and "Show mean differences") will not affect what is displayed in simple regression (since there are no categorical variables in simple regression).

The "Plot Controls" menu allows the user basic control over how the visuals are displayed. The user can change point transparency, jitter variables (about which we will discuss shortly), and alter the plot's theme and color scheme.

Figure 8 shows an example regression/scatterplot. Here, motivation is used to predict health scores. The scatterplot indicates a fairly weak relationship between the two, which is confirmed by the relatively weak slope for motivation (slope $=-0.118, \beta=-0.166$ ).

\section{Modeling quadratic relations}

Flexplot also allows users to model nonlinear relationships using polynomial terms, such as quadratic and cubic relationships. Doing so is a two-step process. First, the user must specify the form of quadratic relationship under the "Visual Fitting” menu item (Regression, Quadratic, or Cubic). This simply tells the module the user wants to fit polynomial terms. It does not, however, tell the program which terms are polynomials. Obviously, with simple regression, only one term can be a polynomial. But, since this module is used for multiple regression as well, this second step is necessary. Figure 9 illustrates how to fit this sort of model.

\section{ANOVA/t test}

When a single categorical variable is entered in the "Independent Variable(s)" box, the software will perform an ANOVA or $t$ test in the background (depending on the number of groups). Flexplot displays the data from ANOVAs/ $t$ tests as (modified) scatterplots. Suppose we have a grouping variable with two levels (e.g., treatment and control). Just as in a linear model, we could convert these groups to numeric variables (e.g., control $=0$ and treatment $=1$ ). Now that we have numeric data, we could plot the data as a scatterplot, as in the left image in Fig. 10. We could even fit a line to the data, and the slope would be equal to the mean difference between groups, while the intercept would equal the mean of the control group (because its $X$ value is zero). However, in this image, there's a lot of overlap between datapoints; all individuals in the treatment group share the same "Group" score (and likewise for those in the control group). With only two unique $X$ values, there's bound to be overlap of $Y$ scores.

To overcome the overlap, and follow the human factors display design principle "make discriminable" (Lee et al., 2017), we can "jitter" the scores. Jittering means we simply add a small amount of random noise to each person's $(0,1)$ score. For example, someone in the control group $($ Group $=0)$ might have a new score of 0.013 , while another person in the control group might have a new score of -0.002 . Both scores are still close to their original value (zero), but that small deviation makes it easier to avoid overlap (middle plot).

Flexplot actually jitters based on density; areas where there's a higher concentration of scores (and thus a higher probability of overlap) are jittered more than areas with lower concentration of scores. These plots, called "bee swarm plots" (Eklund, 2012) are a hybrid between violin plots and simple jittered plots. The advantage of these plots is they convey information about central tendency (via center red dot), spread (via whiskers as well as the raw data), distribution (via the width of the jittering), and the sample size (via the dots). Including information beyond central tendency may increase accuracy and improve conclusions people make (Potter, Kniss, Riesenfeld, \& Johnson, 2010).

To perform an ANOVA (or $t$ test) and generate bee swarm plots, the user only needs to specify a categorical variable as a predictor and the model will generate an appropriate graphic. Also, for nominal (but not ordinal) predictors, Flexplot will sort the $X$-axis by each group's mean for $Y$, where the group with the highest mean is the left-most group, the group with the second highest mean is right of that, etc. Sorting the axis in this way allows users to "chunk" the progression of mean differences into a unit, which will make it easier to remember information than if the ordering of groups on the $X$-axis were arbitrary (Kosslyn, 2006).

The Visual Modeling module will also report means, mean differences, and Cohen's $d$ estimates, as well as confidence intervals. As with regression, users can ask for univariate plots (which will show a histogram for the numeric variable and a bar chart for the categorical variable) and diagnostic plots. Also, they can specify point transparency, as well as the amount of jittering. Flexplot defaults to 0.1 jittering. (This means that the maximum amount of noise added to the numeric score is 0.1 . So, a score of 0 would be, at most 0.1 , and at least-0.1). An example of different amounts of jittering is shown in Fig. 11. 


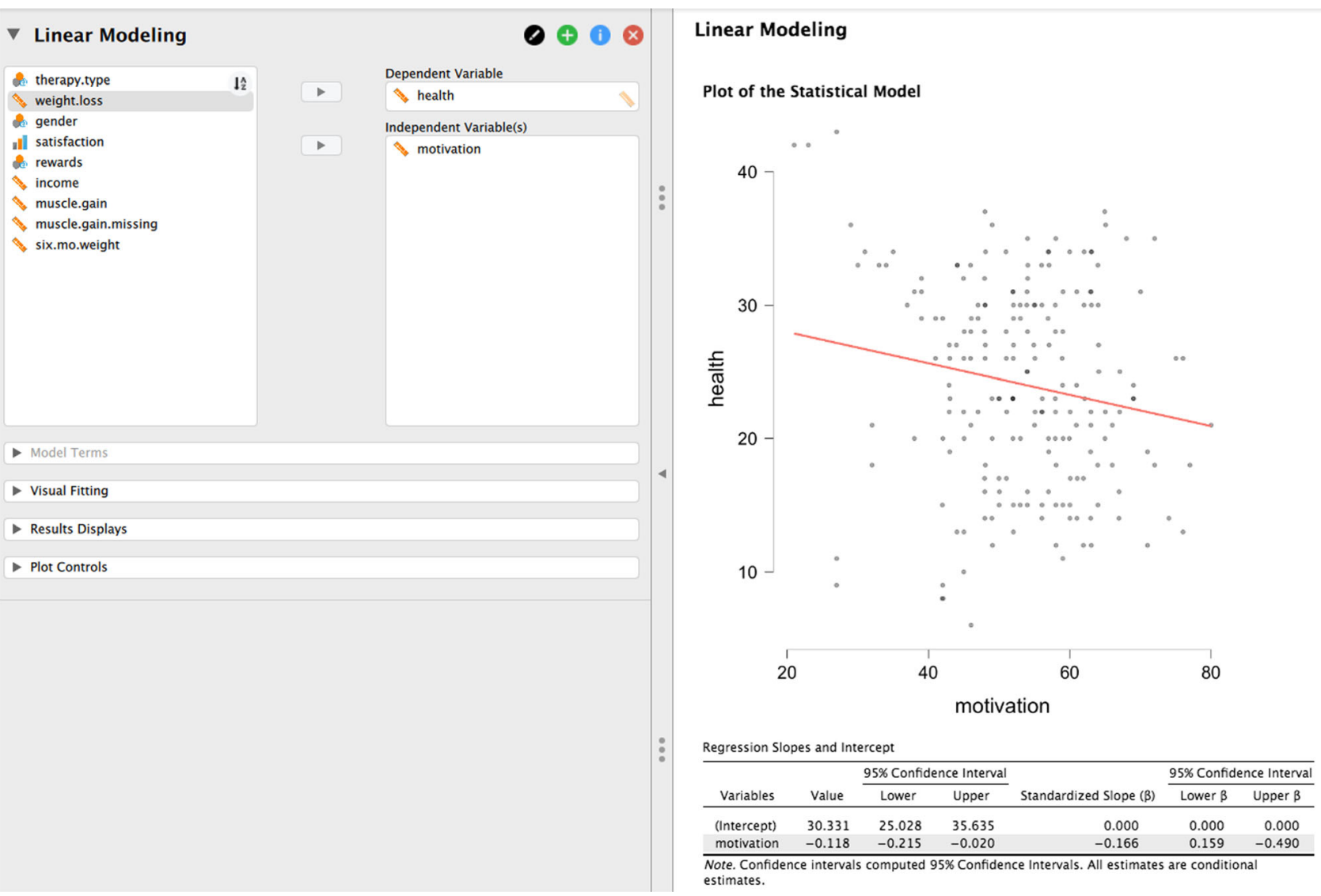

Fig. 8 Simple regression example performed in the Visual Modeling module in JASP

\section{Factorial ANOVA}

Recall that a factorial ANOVA includes two grouping variables (e.g., treatment and gender). Much like with a $t$ test or a regular ANOVA, the general linear model approach to ANOVA will convert the groups to numbers and perform regression. This conversion is more complicated than with a $t$ test (and more complicated than an ANOVA). Fortunately, the computer handles this in the background.

In the software, the user only needs to specify two categorical variables in the "Independent Variable(s)" box to perform a factorial ANOVA. In the background, Flexplot will create

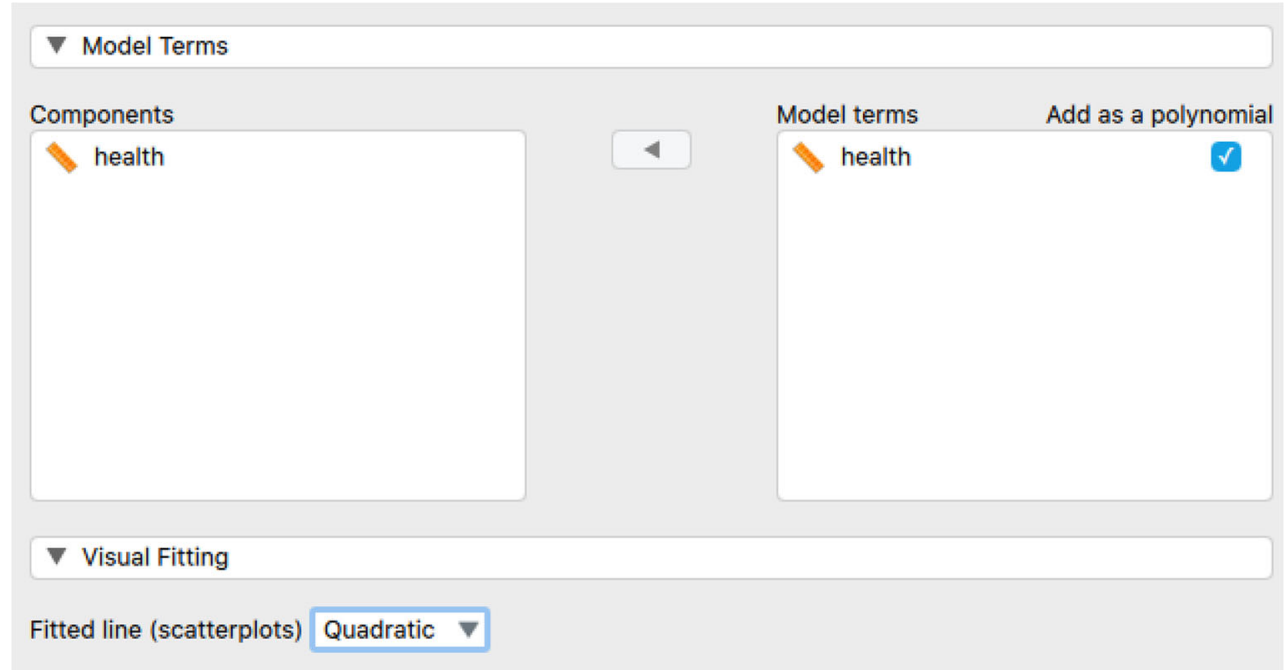

Fig. 9 Illustration of how to model a polynomial regression in JASP. The user must both click on the "Add as a polynomial," checkbox and select "Quadratic" (or "Cubic") from the dropdown menu under the Visual Fitting menu 


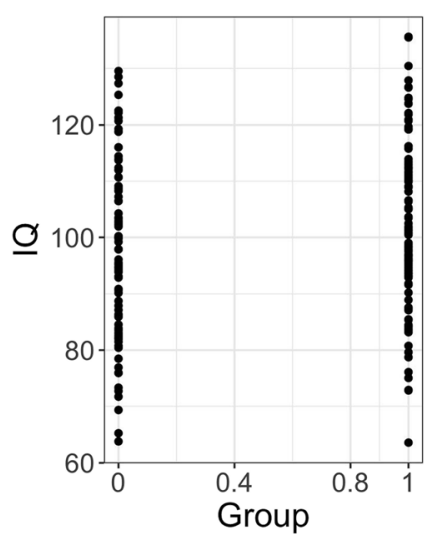

Fig. 10 Illustration of jittering categorical $\mathrm{X}$ variables. The left plot shows a scatterplot of the categorical variable (converted to a number) against the numeric outcome. The middle plot "jitters" the scores by

different bee swarm plots for each level of the second variable entered. For example, if the user enters gender first, then treatment condition, and if treatment condition has three levels (e.g., Placebo, Treatment A, Treatment B), Flexplot will create three panels, one for each condition. The reason Flexplot defaults to panels rather than displaying these as colors/ symbols within the same plot is two-fold: (1) it is harder for humans to detect colors/symbols differences than it is to detect positional differences (Cleveland \& McGill, 1984). Separating into panels maintains positional differences

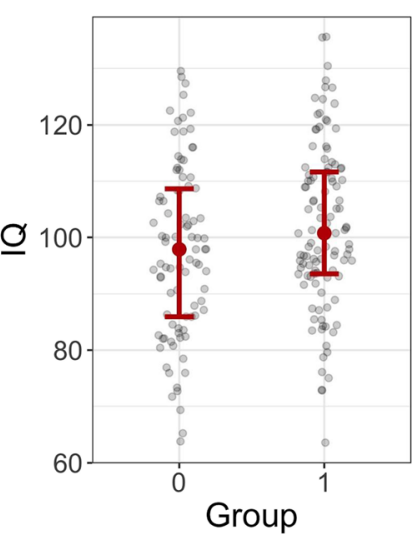

adding random noise to each person's numeric $\mathrm{X}$ value. The right plot jitters the scores as in the middle plot, but the amount of jittering varies based on the density of $\mathrm{Y}$ scores for that particular group

between datapoints without resorting to colors/symbols. (2) Separating these plots into panels minimizes overlap between datapoints. When data overlap, it is harder to encode visual information. Separating the data into panels is consistent with the human factors display design principle to "make discriminable" (Lee et al., 2017). Of course, when data are separated by panels, the eye must travel a further distance to make comparisons across groups, which makes it more difficult to process information. In other words, creating panels violates the HF display principle to "minimize information access cost"

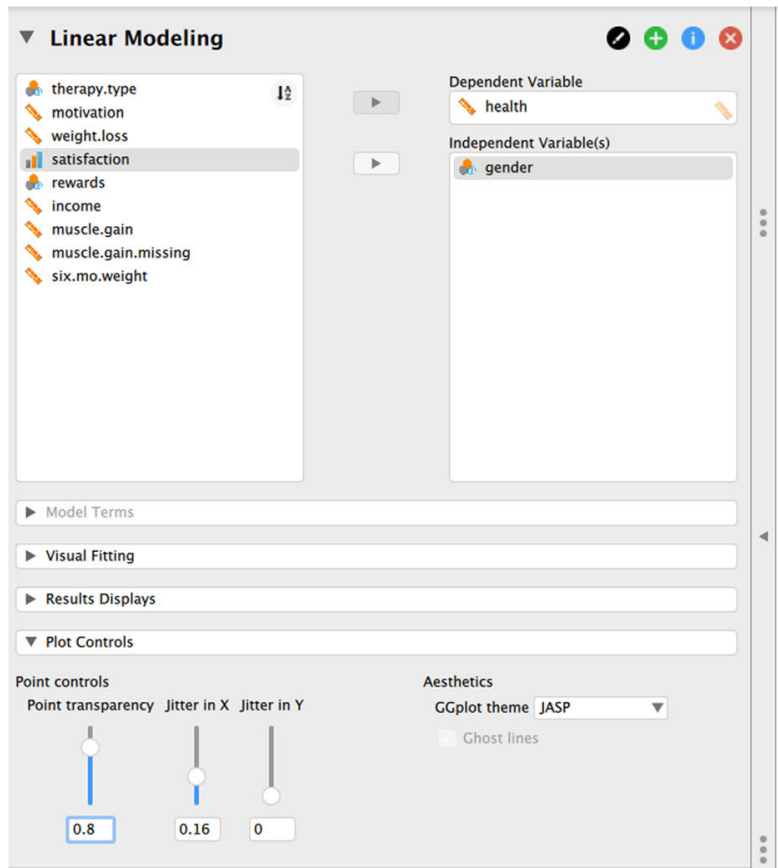

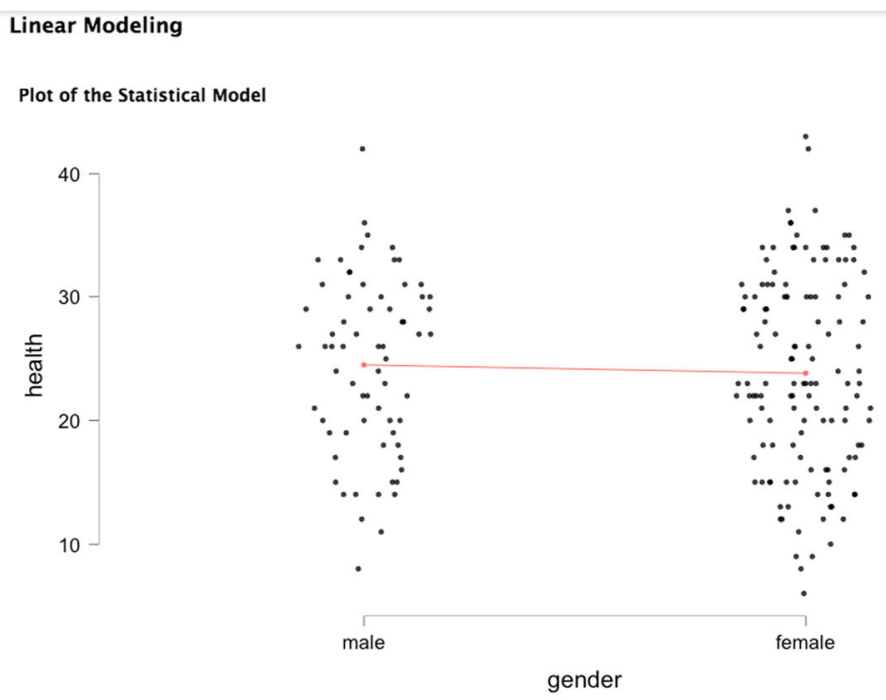

Means of Categorical Variables
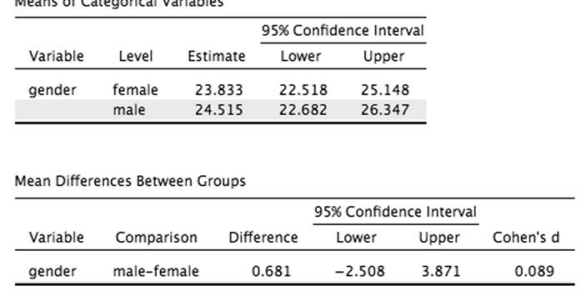

Fig. 11 Demonstration of a $t$ test. The Visual Modeling module will default to producing a graphic of the statistical model, report means, and report mean differences. We have also increased the point transparency (to 0.8) and amount of jittering (to 0.16) 
(Lee et al., 2017). For this reason, Flexplot defaults to displaying ghost lines. The ghost lines serve as "perceptual landmarks" that make it easier for viewers to integrate the information across the more legible panels, consistent with the HF display principle to "support visual momentum," by providing context, a fixed structure (consistent axis ranges), and a common reference (the ghost line). However, the ghost lines can be omitted under the "Plot Controls" menu.

The software will also report means for each level of each variable, as well as mean differences, Cohen's $d$, and confidence intervals. The user also has the option to perform model comparisons. Table 1 shows an example of analysis with a simulated dataset about weight loss. This table reports semipartial $R^{2}$ values. Note that a semi-partial $R^{2}$ states how much the model's $R^{2}$ would reduce if we were to eliminate that parameter from the model. For example, in the table below (reproduced from JASP), the gender semi-partial is very near zero, which means that if we were to eliminate gender from the model, our $R^{2}$ would drop very little. On the other hand, if we dropped rewards, the model's $R^{2}(0.304)$ would drop by 0.299 .

The semi-partial Bayes factors are similar. Bayes factors weigh the evidence in favor of one model over the evidence in favor of the other. The third column weighs the evidence in favor of the reduced model (i.e., the model without that particular term) against the full model. Bayes factors near one indicate there is equal evidence for the two models. For the second column, values much higher than one indicate a variable should stay in the model. If the values are much smaller than one, that variable should be removed. The third column is simply the inverse of the second column. (Sometimes it is easier to interpret Bayes factors higher than one than those less than one). If the "Show $p$ values" button were checked, JASP would also report test statistics, degrees of freedom, and $p$ values.

\section{Modeling interactions}

One salient characteristic of factorial ANOVAs is they allow one to model interactions. Likewise, the Visual Modeling submodule allows users to model interactions. To do so, the

Table 1 Example of estimates obtained from the visual modeling module in JASP

\begin{tabular}{llll}
\hline Term & Semi-partial $R^{2}$ & Semi-partial BF & Inverted BF \\
\hline Full model & 0.304 &. & $\cdot$ \\
gender & 0.000 & 0.071 & 14.134 \\
rewards & 0.299 & $2.418 \mathrm{e}+14$ & $4.135 \mathrm{e}-15$ \\
\hline
\end{tabular}

Note. Semi-partials indicate the effect of removing that particular term from the model. Bayes factors are computed using the BIC user simply enters the "Model Terms" menu, selects the variables they think interact from the "Components" box, then clicks the arrow. Doing so will add interaction terms (see Fig. 12). As before, we can use model comparisons to determine whether it's worth keeping the interaction. Also as before, Flexplot will display visuals that reflect the statistical model. The second variable entered will be displayed in separate panels, and ghost lines will be added to make it easier to compare across panels.

\section{ANCOVA}

An ANCOVA models the relationship between the outcome variable and two predictor variables: one of which is categorical (e.g., treatment group) and the other numeric (i.e., the covariate). To model this in the software, the user only needs to specify a categorical and numeric predictor in the "Independent variable(s)" box and, once again, Flexplot will do the heavy lifting in the background.

Because both numeric and categorical predictors are used, the software will report means/mean differences for the categorical variables, as well as the intercept and slope. The default graphic produced by Flexplot will show the covariate on the $X$-axis, the outcome on the $Y$-axis, and separate panels for each level of the categorical variable. The reason for this is because it results in the least compression of the data, while minimizing overlap. Putting the covariate on the $X$-axis prevents it from being binned, and putting the categorical variable in panels prevents overlap between datapoints. Again, by default, the software will generate ghost lines to make it easier to compare across panels.

\section{Evaluating the homogeneity of regression assumption}

When performing an ANCOVA, the analyst assumes "homogeneity of regression." This simply means the slope between the covariate and the outcome is the same across groups. Put differently, the assumption of homogeneity of regression states there is no interaction between the covariate and the grouping variable. This may or may not be a realistic assumption.

Fortunately, evaluating the assumption is simple. One simply adds an interaction term to the model. Again, this is done by highlighting both variables under the "Components" box and clicking the arrow to move them into the "Model terms" box. The software will automatically update the graphic and the estimates. One can then evaluate the assumption visually (if the lines are not parallel, the assumption has been violated), as well as statistically. To evaluate statistically, we recommend the user click on the "Model comparisons" checkbox in the Results Displays menu. For an ANCOVA, only statistics for the interaction will be reported (as well as the $R^{2}$ for the full model). The reason only the interaction is reported is 


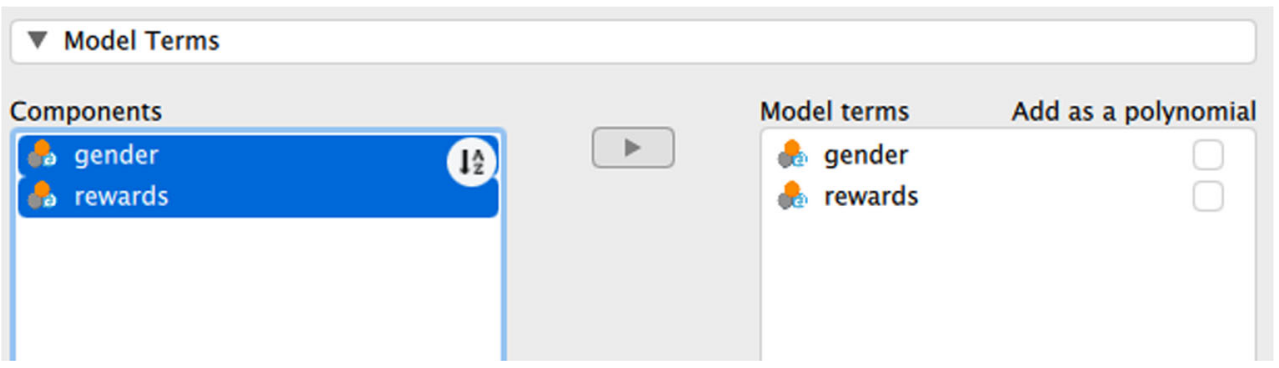

Fig. 12 Example of how to model interactions

because, if there are indeed interactions present in the model, main effect should not be interpreted (Appelbaum \& Cramer, 1974).

As an example, we fit a model predicting weight loss from therapy type, covarying motivation. Table 2 shows the estimates, while Fig. 13 shows the graphics. Although the graphic suggests the lines are not parallel, Table 2 suggests the deviations from parallel are likely just noise; the interaction only adds 0.006 explained variance, and the evidence for the model without the interaction is 95.294 times stronger than the evidence with the interaction.

Added variable plots When researchers conduct an ANCOVA, they are typically interested in assessing mean differences among treatment groups after controlling for the covariate. In this situation, the default graphic produced by Flexplot does not actually reflect the statistical model. Why then does it default to a graphic that does not reflect the statistical model? This graphic allows the user to evaluate the viability of the homogeneity of regression assumption. Once the user is confident the model meets the assumption, a better representation of the statistical model is an added variable plot (AVP; Kutner, Nachtsheim, Neter, \& Li, 2004).

What AVPs do is build a simple regression model between the covariate and the outcome. Subsequently, Flexplot will extract the residuals of that model, then plot the grouping variable against the residuals. The reason for extracting residuals is simple; residuals reflect what remains in the outcome variable after subtracting out the linear effect of the covariate.

Table 2 Results of an ANCOVA Model, Reproduced From JASP

\begin{tabular}{llll}
\hline Term & Semi-partial $R^{2}$ & Semi-partial & Inverted \\
& Bayes factor & Bayes factor \\
\hline
\end{tabular}

Full model $\quad 0.222$

therapy.type

motivation

therapy.type $\times$ motivation $\quad 0.006$

0.010

95.294

Note. Note: Semi-partials indicate the effect of removing that particular term from the model. Bayes factors are computed using the BIC. Main effect estimates of $R^{2}$ and $\mathrm{BF}$ have been suppressed because there is an interaction in the model
Residualizing the effect of the covariate is essentially the same as statistically leveling the playing field on the covariate across all participants. If we then plot the grouping variable against the residuals, this graphic shows the relationship between each condition and the covariate-removed dependent variable.

Aside from representing common statistical models, another primary advantage of AVPs is they simplify the visualization of multivariate relationships. Provided the multivariate models do not contain interaction effects, AVPs can visualize any number of predictor variables using only a simple scatterplot (or bee swarm plot).

However, Flexplot makes one small modification. Technically, residuals have a mean of zero. If the $Y$-axis shows a different scale than the original variables, it can be confusing to those unfamiliar with AVPs. According to Lee et al. (2017), graphical information should "support correct mental models" (p. 188). This principle states that if one knows what sort of background knowledge a user has, we should design with that expertise in mind. Presumably, the user knows the scale of the original variables and, as such, the plots should reflect that. To do so, Flexplot simply adds the mean of the dependent variable back to the residuals.

In the software, the last variable entered will be displayed on the $X$-axis, while the other variable(s) will be residualized from the dependent variable. Because of this, we recommend users input the covariate first, then the grouping variable. The software will then display a bee swarm plot of the groups.

Returning to our previous example, let us now plot an AVP (Fig. 14). Notice the label for the $Y$-axis has changed (from weight.loss to weight.loss $\mid$ motivation), indicating the $Y$-axis displays residuals, rather than the raw weight loss score. Also, the graphic no longer panels, but instead plots bee swarm plots. In JASP, all AVPs will display a simple bivariate relationship.

\section{Multiple regression}

Traditionally, multiple regression refers to any analysis that includes multiple numeric predictor variables. Hopefully, we've made it clear variable type doesn't matter; any two variables, regardless of the permutation of categorical/ 


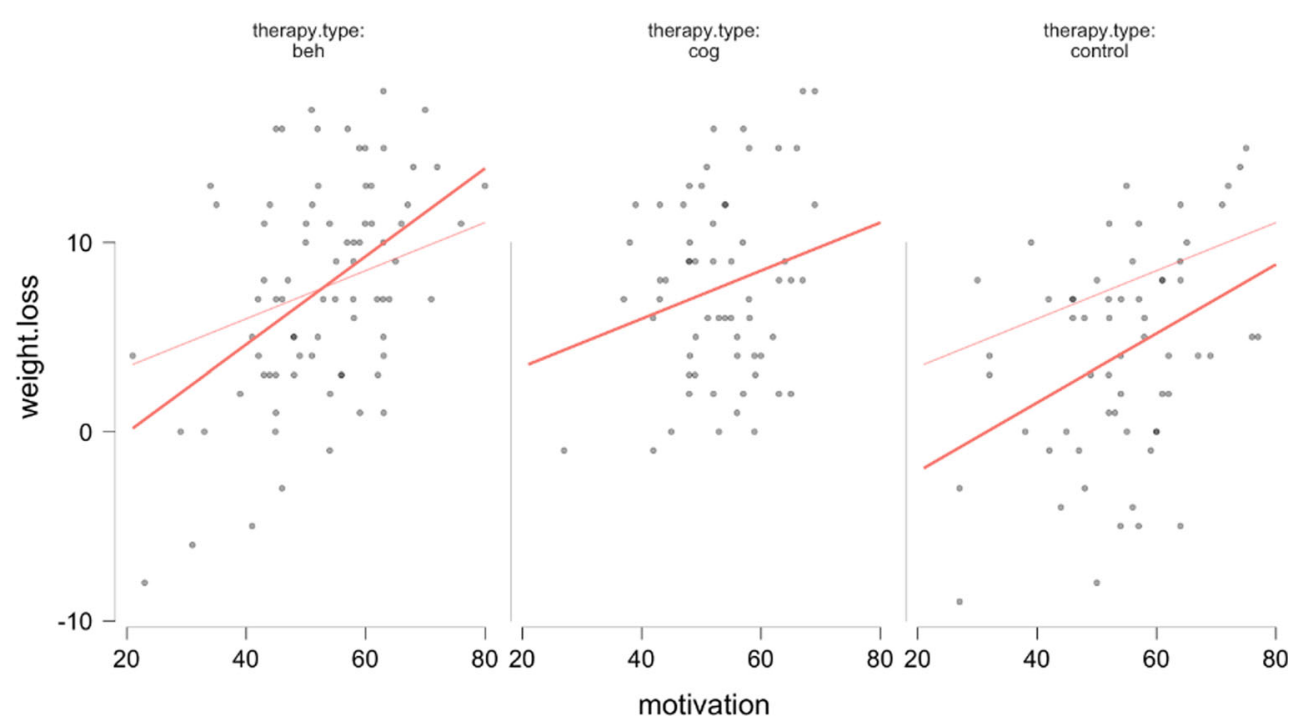

Fig. 13 Default graphic for an ANCOVA model, but modeling the interaction. The nonparallel lines suggest there may be an interaction, but Table 2 suggests otherwise

numeric can be considered multiple regression. And, once again, the software simplifies the process immensely: the user only needs to specify the predictor variables of interest. The computer will then decide how to plot it and what estimates to report.

Many of the tools already introduced can be used for multiple variables, including ghost lines, model comparisons, interaction terms, polynomial terms, etc. With multiple variables, however, the demand on one's visual processing system can be quite taxing. In these situations, AVPs, ghost lines, and transparency can be used to simplify the analysis. For examples of how to simplify complex multivariate visuals, see Fife (2020a).

\section{Other models}

We have covered many of the most popular statistical models researchers might choose to utilize. However, many other models are possible with Flexplot, including mixed models (top image in Fig. 15), logistic regression models (top middle image in Fig. 15), Poisson regression models (top right image in Fig. 15), related $t$ tests (bottom right in Fig. 15), and $\chi^{2}$ models (bottom left in Fig. 15). For more details on fitting these sorts of models, see https://jasp-stats.org/2020/ 04/21/the-visual-modeling-module.

While the goal of Flexplot is to provide intuitive visuals for every possible analysis a user may wish to conduct, doing so

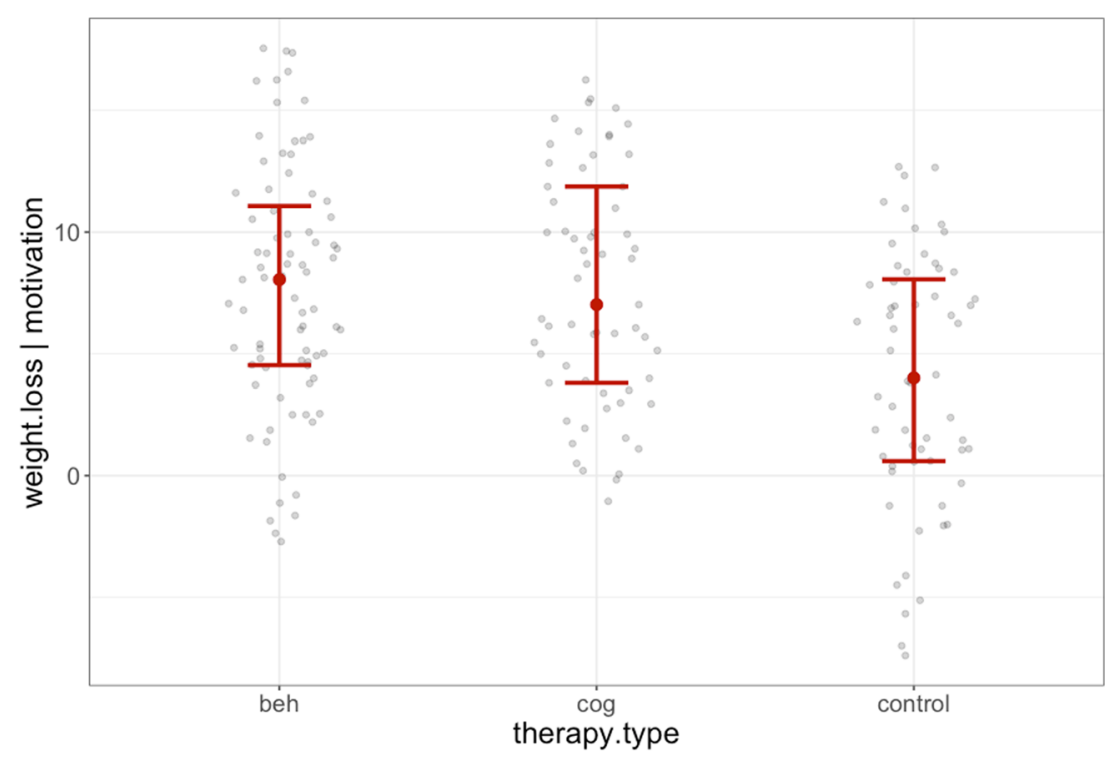

Fig. 14 An added variable plot of the means of the therapy conditions, after controlling for motivation 


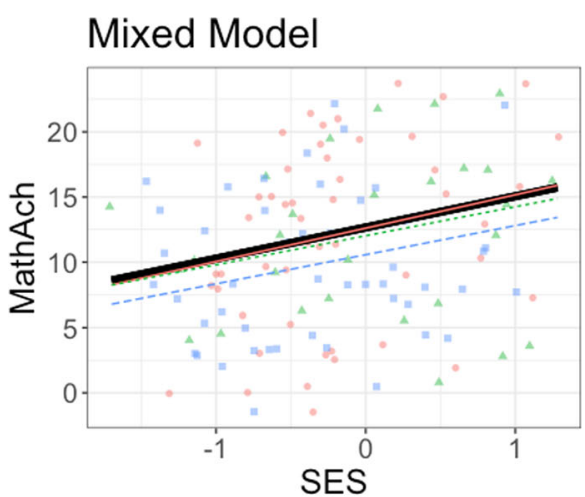

Related T

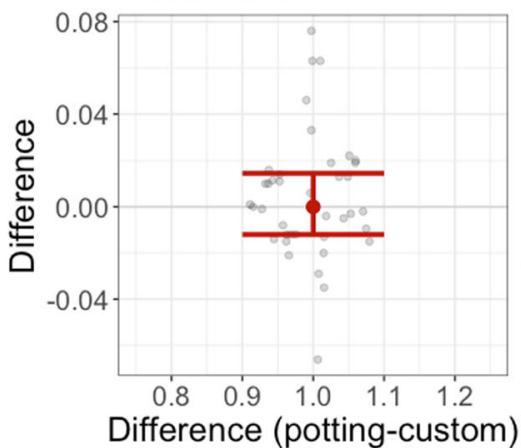

Logistic

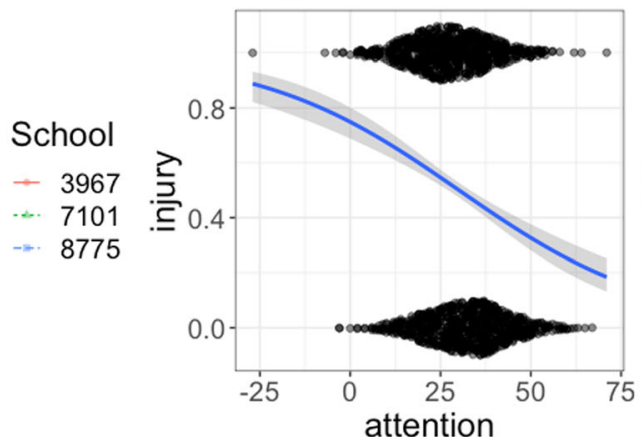

Chi Square

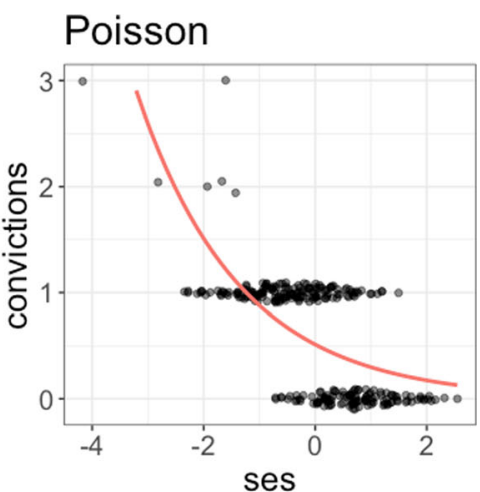

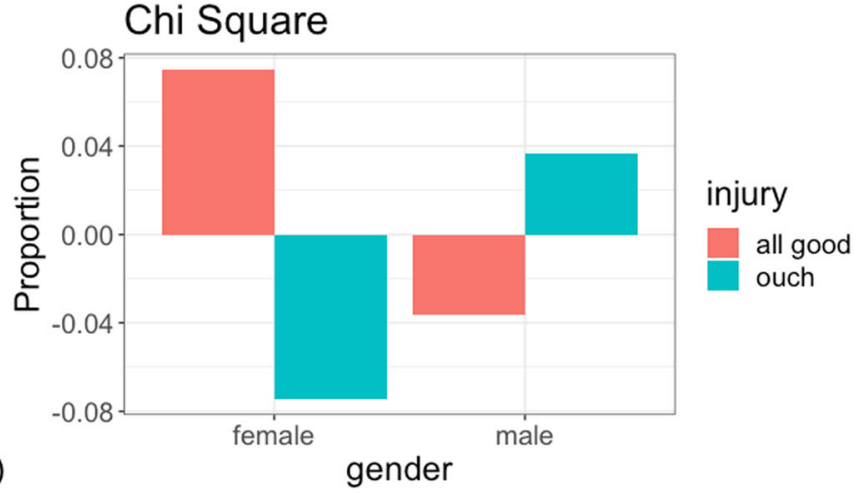

Fig. 15 Additional statistical model graphics available through Flexplot

for some analyses is tricky. Latent variable models, for example, are, by definition, unobserved. Consequently, plotting the data from these models is not intuitive (although, for some examples of visuals others have recommended, see Asparouhov \& Muthén, 2014; Hallgren, McCabe, King, \& Atkins, 2019). Also, analyses are typically performed on summary statistics (e.g., covariances), making plots of raw data more difficult. In other words, while Flexplot aims to provide visuals for all analyses, as of yet, visuals for certain types of models (factor analysis, structural equation modeling, time series analyses) have yet to be developed, though this will change over time.

\section{Discussion}

Methodological revolutions of the past (e.g., the computational revolution, as well as the first push against significance testing) have come and gone, with little to show in terms of sound data visualization practices. Most graphics in scientific research utilize suboptimal graphics that exacerbate visualization biases (Pastore, Lionetti, \& Altoè, 2017; Weissgerber et al., 2015; Zacks \& Tversky, 1999). In this paper, we argue existing software defaults have exacerbated the problem and increased analysts' tolerance for bad visuals; either existing software cannot produce sound graphics, or creating these sorts of visuals requires a great deal of effort that promotes visualization-free analyses.
In this paper, we promote the use of Flexplot, which is available in R, Jamovi, and JASP (via the Visual Modeling module). Flexplot offers several advantages: first, its visuals were designed based on principles of human perception. Second, Flexplot automates much of the data visualization, including producing visuals alongside statistical analyses. This automation makes it far more likely researchers will utilize the graphics to aid in quality control, model evaluation, and data interpretation.

In this paper, we have demonstrated how to perform many of the most common analyses, including t-tests, ANOVAs, ANCOVAs, factorial ANOVAs, and multiple regression. Flexplot is also capable of visualizing more complex models, including Mixed Models and logistic regressions. ${ }^{7}$ While visualization tools for some models are limited, this will change in the near future.

Admittedly, visuals alone will not address the current upheaval. If, as some argue, the current crisis was primarily caused by questionable research practices (Fife \& Rodgers,

\footnotetext{
${ }^{7}$ Very often, any model that utilizes the predict method in R can be visualized with Flexplot. Flexplot generates predicted values for statistical models by first creating a dataset of predictors, each of which spans the entire range of the predictors. For example, if a model has "male" and "female" as one predictor and stress as another (ranging from, say, 1 to 10), Flexplot will create a dataset that crosses male and female with 30 (the default) stress scores, equally spaced, from 1 to 10 . This will generate a dataset with $30 \times 2=60$ observations. This is to ensure the predicted dataset covers the entire range of the data. Then, for each of those 60 observations, Flexplot will feed the statistical model the observations and estimate predicted values.
} 
2019; Shrout \& Rodgers, 2018), plotting a graphic will not resolve that. Analysts can still p-hack, hypothesize after results are known (HARK), and fabricate data. However, we do think greater reliance on visualization in scientific judgment is critical. Visuals can highlight problems with statistical models, preventing spurious publications and the perpetuation of canonized falsehoods (Nissen, Magidson, Gross, \& Bergstrom, 2016). Visuals also present raw data in their entirety, which allows readers to gauge the credibility of the results themselves (Tay, Parrigon, Huang, \& LeBreton, 2016), rather than relying on arbitrary thresholds of probability. Finally, visuals allow users to easily understand statistical models. Even untrained users can make sophisticated statistical decisions based on graphics alone (Correll, 2015; Correll \& Gleicher, 2014; Szafir, Haroz, Gleicher, \& Franconeri, 2016). It is interesting to consider how many important insights might have been missed because researchers failed to visualize their data. It is our hope that this article provides a first step towards preventing these sorts of missed insights.

\section{References}

Adams, J. A. (1989). Human factors engineering. New York, NY: Macmillan Publishing Co, Inc.

Anscombe, F. J. (1973). Graphs in statistical analysis. American Statistician, 27(1), 17-21. https://doi.org/10.1080/00031305.1973. 10478966

Appelbaum, M. I., \& Cramer, E. M. (1974). Some problems in the nonorthogonal analysis of variance. Psychological Bulletin, 81(6), 335.

Asparouhov, T., \& Muthén, B. O. (2014). Using Mplus individual residual plots for diagnostics and model evaluation in SEM, (20), 1-14.

Baddeley, A. (1994). The Magical Number Seven: Still Magic After All These Years? Psychological Review, 101(2), 353-356. https://doi. org/10.1037/0033-295x.101.2.353

Bertin, J. (2010). Semiology of graphics: Diagrams, networks, maps. Redlands, CA: Esri Press.

Butler, D. L. (1993). Graphics in psychology: Pictures, data, and especially concepts. Behavior Research Methods, Instruments, \& Computers (Vol. 25).

Card, S. (2009). Information visualization. In A. Sears \& J. A. Jacko (Eds.), Human-computer interaction: Design issues, solutions, and applications (pp. 181-216). Boca Raton, Florida: CRC Press.

Chernoff, H. (1973). The use of faces to represent points in k-dimensional space graphically. Journal of the American Statistical Association, 68(342), 361-368.

Cleveland, W. S., \& McGill, R. (1984). Graphical perception: Theory, experimentation, and application to the development of graphical methods. Journal of the American Statistical Association, 79(387), $531-554$.

Cohen, J. (1968). Multiple regression as a general data-analytic system. Psychological Bulletin, 426-443. Retrieved from http://citeseerx.ist. psu.edu/viewdoc/summary?doi=10.1.1.476.6180

Correll, M. A. (2015). Visual Statistics. University of WisconsinMadison.

Correll, M. A., \& Gleicher, M. (2014). Error Bars Considered Harmful: Exploring Alternate Encodings for Mean and Error. IEEE
Transactions on Visualization and Computer Graphics, 20(12), 2142-2151. https://doi.org/10.1109/TVCG.2014.2346298

Correll, M. A., Li, M., Kindlmann, G., \& Scheidegger, C. (2019). Looks Good to Me: Visualizations As Sanity Checks. IEEE Transactions on Visualization and Computer Graphics, 25(1), 830-839. https:// doi.org/10.1109/TVCG.2018.2864907

Counsell, A., \& Harlow, L. L. (2017). Reporting practices and use of quantitative methods in Canadian journal articles in psychology. Canadian Psychology/Psychologie Canadienne, 58(2), 140-147. https://doi.org/10.1037/cap0000074

Cumming, G., Fidler, F., Leonard, M., Kalinowski, P., Christiansen, A., Kleinig, A., ... Wilson, S. (2007). Statistical Reform in Psychology Is Anything Changing ? Psychological Science, 18(3), 1-4. https:// doi.org/10.1111/j.1467-9280.2007.01881.x

Dimara, E., Franconeri, S., Plaisant, C., Bezerianos, A., \& Dragicevic, P. (2018). A task-based taxonomy of cognitive biases for information visualization. IEEE Transactions on Visualization and Computer Graphics

Eklund, A. (2012). Beeswarm: the bee swarm plot, an alternative to stripchart. R Package Version 0.1, 5 .

Fife, D. A. (2020a). Flexplot: Graphical-Based Data Analysis. PsyArxiv. $10.31234 / \mathrm{osf} . \mathrm{io} / \mathrm{kh} 9 \mathrm{c} 3$

Fife, D. A. (2020b). The Eight Steps of Data Analysis: A Graphical Framework to Promote Sound Statistical Analysis. Perspectives on Psychological Science, 15(4), 1054-1075. https://doi.org/10.1177/ 1745691620917333

Fife, D., \& Rodgers, J. L. (2019). Exonerating EDA, Expanding CDA: A Pragmatic Solution to the Replication Crisis. https://doi.org/10. 31234/osf.io/5vfq6

Gigerenzer, G. (2004). Mindless statistics. The Journal of SocioEconomics, 33(5), 587-606. https://doi.org/10.1016/J.SOCEC. 2004.09.033

Hallgren, K. A., McCabe, C. J., King, K. M., \& Atkins, D. C. (2019). Beyond path diagrams: Enhancing applied structural equation modeling research through data visualization. Addictive Behaviors, 94, 74-82. https://doi.org/10.1016/j.addbeh.2018.08.030

Hansen, C. D., Chen, M., Johnson, C. R., Kaufman, A. E., \& Hagen, H. (2014). Scientific Visualization: Uncertainty, Multifield, Biomedical, and Scalable Visualization. London: Springer. Retrieved from http://www.springer.com/series/4562

Healey, C. G., Booth, K. S., \& Enns, J. T. (1996). High-speed visual estimation using preattentive processing. ACM Transactions on Computer-Human Interaction (TOCHI), 3(2), 107-135.

Healy, K., \& Moody, J. (2014). Data Visualization in Sociology. Annual Review of Sociology, 40(1), 105-128. https://doi.org/10.1146/ ANNUREV-SOC-071312-145551

Hofman, J. M., Goldstein, D. G., \& Hullman, J. (2020). How Visualizing Inferential Uncertainty Can Mislead Readers About Treatment Effects in Scientific Results. https://doi.org/10.1145/3313831. 3376454

Hollands, J. G., \& Spence, I. (1998). Judging Proportion with Graphs: The Summation Model. Applied Cognitive Psychology, 12(2), 173190. https://doi.org/10.1002/(SICI)1099-0720(199804)12:2<173:: AID-ACP499>3.0.CO;2-K

Hu, K., Orghian, D., \& Hidalgo, C. (2018). DIVE: A mixed-initiative system supporting integrated data exploration workflows. Proceedings of the Workshop on Human-In-the-Loop Data Analytics, HILDA 2018. https://doi.org/10.1145/3209900.3209910

Inbar, O., Tractinsky, N., \& Meyer, J. (2007). Minimalism in information visualization: attitudes towards maximizing the data-ink ratio. In ECCE (Vol. 7, pp. 185-188).

JASP Team. (2019). JASP (Version 0.10.2)[Computer software]. Retrieved from https://jasp-stats.org/

Jun, E., Daum, M., Roesch, J., Chasins, S., Berger, E., Just, R., \& Reinecke, K. (2019). Tea: A High-level Language and Runtime System for Automating Statistical Analysis. In Proceedings of the 
32nd Annual ACM Symposium on User Interface Software and Technology (pp. 591-603).

Kale, A., Kay, M., \& Hullman, J. (2020). Visual Reasoning Strategies for Effect Size Judgments and Decisions. Retrieved from http://arxiv. org/abs/2007.14516

Kandel, S., Parikh, R., Paepcke, A., Hellerstein, J. M., \& Heer, J. (2012). Profiler: Integrated statistical analysis and visualization for data quality assessment. In Proceedings of the Workshop on Advanced Visual Interfaces AVI (pp. 547-554). New York, New York, USA: ACM Press. https://doi.org/10.1145/2254556.2254659

Kantowitz, B. H. (1987). 3. Mental workload. In Advances in psychology (Vol. 47, pp. 81-121). Elsevier.

Kohn, L. T., Corrigan, J., \& Donaldson, M. S. (2000). To err is human: building a safer health system (Vol. 6). National academy press Washington, DC.

Kosslyn, S. M. (2006). Graph Design for Eye and Mind. New York, New York, USA: Oxford University Press. https://doi.org/10.1016/B9781-4557-7896-6.00058-3

Kutner, M. H., Nachtsheim, C. J., Neter, J., \& Li, W. (2004). Applied linear statistical models. Applied linear statistical models. New York, NY: McGraw-Hill/Irwin.

Kyonka, E. G. E., Mitchell, S. H., \& Bizo, L. A. (2019). Beyond inference by eye: Statistical and graphing practices in JEAB, 1992-2017. Journal of the Experimental Analysis of Behavior, 111(2), 155165. https://doi.org/10.1002/jeab.509

Lee, M. D., Blanco, G., \& Bo, N. (2017). Testing take-the-best in new and changing environments. Behavior Research Methods, 49(4), 1420-1431. https://doi.org/10.3758/s13428-016-0798-x

Lee, M. D., Reilly, R. E., \& Butavicius, M. E. (2003). An empirical evaluation of Chernoff faces, star glyphs, and spatial visualizations for binary data. In Proceedings of the Asia-Pacific symposium on Information visualisation-Volume 24 (pp. 1-10).

Levine, S. S. (2018). Show us your data: Connect the dots, improve science. Management and Organization Review, 14(2), 433-437. https://doi.org/10.1017/mor.2018.19

Mackinlay, J. (1986). Automating the Design of Graphical Presentations of Relational Information. ACM Transactions on Graphics (TOG), 5(2), 110-141. https://doi.org/10.1145/22949.22950

Mackinlay, J., Hanrahan, P., \& Stolte, C. (2007). Show me: Automatic presentation for visual analysis. IEEE Transactions on Visualization and Computer Graphics, 13(6), 1137-1144.

Matejka, J., \& Fitzmaurice, G. (2017). Same stats, different graphs: generating datasets with varied appearance and identical statistics through simulated annealing. In Proceedings of the 2017 CHI Conference on Human Factors in Computing Systems (pp. 1290-1294).

Miller, G. A. (1956). The magical number seven, plus or minus two: Some limits on our capacity for processing information. Psychological Review, 63(2), 81.

Moritz, D., Wang, C., Nelson, G. L., Lin, H., Smith, A. M., Howe, B., \& Heer, J. (2018). Formalizing visualization design knowledge as constraints: Actionable and extensible models in draco. IEEE Transactions on Visualization and Computer Graphics, 25(1), 438-448.

Muenchen, B. (2019). Is Scholarly Use of R Beating SPSS Already? r4stats.com. Retrieved October 6, 2020, from http://r4stats.com/ 2019/07/15/is-scholarly-use-of-r-use-beating-spss-already/

Munzner, T. (2014). Visualization analysis and design. CRC press.

Newman, G. E., \& Scholl, B. J. (2012). Bar graphs depicting averages are perceptually misinterpreted: The within-the-bar bias. Psychonomic Bulletin \& Review, 19(4), 601-607.

Nissen, S. B., Magidson, T., Gross, K., \& Bergstrom, C. T. (2016). Publication bias and the canonization of false facts. ELife, 5(e21451). https://doi.org/10.7554/eLife.21451

Norman, D. (2014). Things that make us smart: Defending human attributes in the age of the machine. Diversion Books.
Oakes, M. (1986). Statistical inference: A commentary for the social and behavioral sciences. New York, NY, USA: John Wiley \& Sons.

Pandey, A. V., Rall, K., Satterthwaite, M. L., Nov, O., \& Bertini, E. (2015). How deceptive are deceptive visualizations?: An empirical analysis of common distortion techniques. In Proceedings of the 33rd Annual ACM Conference on Human Factors in Computing Systems (pp. 1469-1478). ACM.

Pastore, M., Lionetti, F., \& Altoè, G. (2017). When one shape does not fit all: A commentary essay on the use of graphs in psychological research. Frontiers in Psychology. Frontiers Media S.A. https:// doi.org/10.3389/fpsyg.2017.01666

Peden, B. F., \& Hausmann, S. E. (2000). Data Graphs in Introductory and Upper Level Psychology Textbooks: A Content Analysis. Teaching of Psychology, 27(2), 93-97. https://doi.org/10.1207/ S15328023TOP2702 03

Potter, K., Kniss, J., Riesenfeld, R., \& Johnson, C. R. (2010). Visualizing summary statistics and uncertainty. In Computer Graphics Forum (Vol. 29, pp. 823-832). Oxford England: Wiley Online Library.

Rodgers, J. L. (2010). The epistemology of mathematical and statistical modeling: a quiet methodological revolution. The American Psychologist, 65(1), 1-12. https://doi.org/10.1037/a0018326

Schild, A. H. E., \& Voracek, M. (2013). Less is less: A systematic review of graph use in meta-analyses. - PsycNET. Research Synthesis Methods, 4(3), 209-219. Retrieved from https://psycnet.apa.org/ record/2013-34734-001

Schmidt, F. L. (1996). Statistical significance testing and cumulative knowledge in psychology: Implications for training of researchers. Psychological Methods, 1(2), 115-129. https://doi.org/10.1037/ 1082-989X.1.2.115

Shrout, P. E., \& Rodgers, J. L. (2018). Psychology, Science, and Knowledge Construction: Broadening Perspectives from the Replication Crisis. Annual Review of Psychology, 69(1), 487-510. https://doi.org/10.1146/annurev-psych-122216-011845

Szafir, D. A., Haroz, S., Gleicher, M., \& Franconeri, S. (2016). Four types of ensemble coding in data visualizations. Journal of Vision, 16(5), 11.

Tay, L., Parrigon, S., Huang, Q., \& LeBreton, J. M. (2016). Graphical Descriptives: A Way to Improve Data Transparency and Methodological Rigor in Psychology. Perspectives on Psychological Science, 11(5), 692-701. https://doi.org/10.1177/ 1745691616663875

The Jamovi Project. (2019). Jamovi (Version 0.9) [Computer Software]. Retrieved from https://www.jamovi.org

Tufte, E. R., \& Graves-Morris, P. R. (1983). The visual display of quantitative information (Vol. 2). Graphics press Cheshire, CT.

Tyron, W. W. (1998). The inscrutable null hypothesis. American Psychologist, 53(7), 796-796. https://doi.org/10.1037/0003-066X. 53.7.796.b

Vicente, K. (2010). The human factor: revolutionizing the way we live with technology. Vintage Canada.

Wainer, H. (2010). Prelude. In J. Berkson (Ed.), Semiology of Graphics: Diagrams, Networks, Maps (2nd ed., pp. ix-x). Redlands, CA: ESRI Press.

Ware, C. (2019). Information visualization: perception for design. Morgan Kaufmann.

Weissgerber, T. L., Milic, N. M., Winham, S. J., \& Garovic, V. D. (2015). Beyond bar and line graphs: time for a new data presentation paradigm. PLoS Biology, 13(4), e1002128-e1002128. https://doi.org/ 10.1371/journal.pbio.1002128

Wickens, C. D. (2008). Multiple resources and mental workload. Human Factors, 50(3), 449-455.

Wickens, C. D. (2014). Effort in human factors performance and decision making. Human Factors, 56(8), 1329-1336.

Wickham, H. (2010). A Layered Grammar of Graphics. Journal of Computational and Graphical Statistics, 19(1), 3-28. https://doi. org/10.1198/jcgs.2009.07098 
Wickham, H. (2016). ggplot2: Elegant Graphics for Data Analysis. Springer-Verlag New York. Retrieved from https://ggplot2. tidyverse.org

Wilkinson, L., \& Task Force on Statistical Inference. (1999). Statistical Methods in Psychology Journals: Guidelines and Explanations. American Psychologist, 54(8), 594-601.

Zacks, J., \& Tversky, B. (1999). Bars and lines: A study of graphic communication. Memory and Cognition, 27(6), 1073-1079. https://doi.org/10.3758/BF03201236
Open Practices Statement

The Flexplot software is available at http://www.github.com/ dustinfife/flexplot

Publisher's note Springer Nature remains neutral with regard to jurisdictional claims in published maps and institutional affiliations. 